\title{
Systematics of 'lithistid' tetractinellid demosponges from the Tropical Western Atlantic - implications for phylodiversity and bathymetric distribution
}

\author{
Astrid Schuster ${ }^{1,2}$, Shirley A. Pomponi ${ }^{3}$, Andrzej Pisera 4 , Paco \\ Cárdenas $^{5}$, Michelle Kelly ${ }^{6}$, Gert Wörheide ${ }^{1,7,8}$, and Dirk Erpenbeck ${ }^{1,8}$ \\ ${ }^{1}$ Department of Earth- \& Environmental Sciences, Palaeontology and Geobiology, \\ Ludwig-Maximilians-Universität München, Richard-Wagner Str. 10, 80333 Munich, \\ Germany \\ ${ }^{2}$ Current address: Department of Biology, NordCEE, Southern University of Denmark, \\ Campusvej 55, 5300 M Odense, Denmark \\ ${ }^{3}$ Harbor Branch Oceanographic Institute, Florida Atlantic University, 5600 U.S. 1 North, \\ Ft Pierce, FL 34946, USA \\ ${ }^{4}$ Institute of Paleobiology, Polish Academy of Sciences, ul. Twarda 51/55, 00-818 \\ Warszawa, Poland \\ ${ }^{5}$ Pharmacognosy, Department of Medicinal Chemistry, Uppsala University, Husargatan \\ 3, 75123 Uppsala, Sweden \\ ${ }^{6}$ National Centre for Coasts and Oceans, National Institute of Water and Atmospheric \\ Research, Private Bag 99940, Newmarket, Auckland, 1149, New Zealand \\ ${ }^{7}$ SNSB-Bayerische Staatssammlung für Paläontologie und Geologie, Richard-Wagner \\ Str. 10, 80333 Munich, Germany \\ ${ }^{8}$ GeoBio-CenterLMU, Ludwig-Maximilians-Universität München, Richard-Wagner Str. 10, \\ 80333 Munich, Germany
}

Corresponding author:

Dirk Erpenbeck ${ }^{1,8}$

Email address: erpenbeck@Imu.de 
Background Among all present demosponges, lithistids represent a polyphyletic group with exceptionally well preserved fossils dating back to the Cambrian. Knowledge of their recent diversity, particularly in the Tropical Western Atlantic Ocean (TWA) where they are common in deep waters, is scarce making any comparison between present and past major 'lithistid' faunas difficult. In addition, the lack of sufficient molecular and morphological data hamper any predictions on phylogenetic relationships or phylodiversity from this region. The Harbor Branch Oceanographic Institute (HBOI, Fort Pierce, Florida) holds the largest collection of TWA lithistid sponges worldwide, however, the majority remain to be taxonomically identified and revised.

Methods/Principal Findings In this study we provide sequences of 249 lithistid demosponges using two independent molecular markers (28S rDNA (C1-D2) and cox1 mtDNA). In addition, a morphological documentation of 70 lithistid specimens is provided in the database of the Sponge Barcoding Project (SBP). This integrated dataset represents the largest and most comprehensive of the TWA lithistids to date. The phylogenetic diversity of 'lithistid' demosponges in the Bahamas and Jamaica are high in comparison to other TWA regions; Theonellidae and Corallistidae dominate the fauna, while Neopeltidae and Macandrewiidae are rare. A new tetractinellid suborder, one new genus and several new species are recognized and the Pacific 'lithistid' genera, Herengeria and Awhiowhio, are reported from the TWA for the first time. The higher-taxa relationships of desma-bearing tetractinellids are discussed and topics for revision suggested.

Conclusion This first integrative approach of TWA 'lithistid' demosponges contributes to a better understanding of their phylogenetic affinities, diversity and bathymetric distribution patterns within the TWA. As in the Pacific, the TWA 'lithistid' demosponges dominate deep-water habitats. Deeper taxonomic investigations will undoubtedly contribute to a better comparison between present major 'lithistid' faunas and their fossil record in the Mesozoic.

\section{INTRODUCTION}

Among all present demosponges, lithistids represent a palaeontologically important polyphyletic group, with exceptionally well preserved fossils dating back to the Cambrian (e.g. Pisera, 2002; 2006), and several relict genera represented in living faunas today (e.g. Lévi, 1991; Pisera, 2002; Kelly, 2007; Kelly et al., 2003). Several key 'lithistid' demosponge faunas are relatively well known: 1) 'lithistid' demosponges are dominant components of seamount communities on the Norfolk Ridge and in the South-West Pacific (e.g. Lévi, 1991; Kelly, 2000, 2007; SchlacherHoenlinger, Pisera and Hooper, 2005; Kelly et al., 2007), and their inventory, morphological identification and molecular systematics has been the focus of several studies (e.g. SchlacherHoenlinger, Pisera and Hooper, 2005; Schuster et al., 2015); 2) large 'lithistid' assemblages are reported from continental shelves and caves of the North-East Atlantic (e.g. Carvalho, Pomponi and Xavier, 2015), and from seamounts in the Mediterranean (e.g. Maldonado et al., 2015).

However, the present-day lithistid species and their phylogenetic diversity in several marine bioregions including the Western Indian Ocean, Subantarctic regions including South Africa, Northern Pacific and Tropical Western Atlantic (TWA) are incompletely understood. While 'lithistid' demosponges in the TWA are reported from continental shelves, caves and slopes by Van Soest and Stentoft (1988), Reed and Pomponi (1997), and Pomponi et al., (2001), and many earlier reports of individual species (e.g. Sollas, 1888), the fauna is still poorly known with few descriptions and no molecular data. This greatly limits the understanding of their phylogenetic relationships, diversity and evolution.

Desma-bearing demosponges, historically referred to as 'lithistid' demosponges, form a polyphyletic group. Molecular systematics now group the majority of 'lithistid' demosponges (11 out of 13 families) to the order Tetractinellida Marshall, 1876. Eight of these families are 
assigned to the suborder Astrophorina Sollas, 1887 and three to the suborder Spirophorina sensu Morrow and Cárdenas 2015 (Cárdenas et al., 2011; Morrow and Cárdenas, 2015; Schuster et al., 2015). Schuster et al. (2015) showed several 'lithistid' families such as Pleromidae Sollas, 1888, Desmanthidae Topsent, 1894 and Scleritodermidae Sollas, 1888 to be polyphyletic, and Corallistidae Sollas, 1888, Theonellidae von Lendenfeld, 1903 and Phymatellidae Schrammen, 1910 to be monophyletic. However, the systematic affinities for families such as e.g. Siphonidiidae von Lendenfeld, 1903, Azoricidae Sollas, 1888 and Neopeltidae Sollas, 1888, remain obscure due to few molecular data available, and hence, only 21 out of 40 'lithistid' genera were evaluated in Schuster et al. (2015). The same study indicated that several spicule types convergently evolved within this sponge group. The families Scleritodermidae and Siphonidiidae were suggested to form a separate clade within Tetractinellida, but outside the two suborders Astrophorina and Spirophorina (Kelly Borges and Pomponi, 1994; Schuster et al., 2015). With the discovery and description of a new tetractinellid family Stupendidae Kelly and Cárdenas, 2016, a sister group relationship of Stupendidae to a clade consisting of rhizomorine-desma-bearing Scleritodermidae, Siphonidiidae and Azoricidae Sollas, 1888 was recently indicated (Kelly and Cárdenas, 2016). However, understanding the higher taxonomic relationships within Tetractinellida including its lithistid lineages is still hindered by incomplete taxon sampling and sequencing of key taxa such as Thrombidae Sollas 1888 or Gastrophanella Schmidt, 1879 (e.g. Kelly Borges and Pomponi, 1994; Cárdenas et al., 2011).

Aside from the report of 'lithistids' in some specific island regions of the TWA, such as Barbados (Van Soest and Stentoft, 1988), the Bahamas (Maldonado and Young, 1996; Reed and Pomponi, 1997), Cuba (Pisera, 1999) the deep Florida shelf (Pisera and Pomponi, 2015), and chemotaxonomic studies (Kelly-Borges et al. 1994) the most comprehensive taxonomy based survey comprising nearly all island groups in the TWA was conducted by Pomponi et al. (2001). The main focus of a study of Pomponi et al. (2001) was the documentation of biodiversity and bathymetric distributions of 'lithistids', thus no morphological species descriptions, sequences or phylogenetic affinities of these specimens were included. Although Pomponi et al. (2001) concluded that 'lithistids' are an important and dominant group of deep, hard-bottom habitats in the TWA, no comprehensive integrative taxonomic approach using molecular and morphological data has yet been made to evaluate this large and unique collection of TWA 'lithistid' demosponges, which is to a large extent unidentified and awaits taxonomic revision. Their study was based on 36 expeditions and 450 submersible transects led by the Harbor Branch Oceanographic Institute (HBOI) from 1984 to 2000, and aimed to provide an inventory of the biodiversity and bathymetric distribution of TWA 'lithistids'. As a result, 28 'lithistid' species representing 18 genera and 9 families were reported from the TWA. However, knowledge of the TWA 'lithistid' fauna still remains comparatively poorly known, but crucial for a better knowledge of their global diversity and their comparison to the Mesozoic 'lithistids'.

The present study presents the first molecular systematic attempt to evaluate a large part of the extensive HBOI 'lithistid' collection $(\approx 250$ specimens) by means of generating independent molecular markers (cox1 and 28S, C1-D2 region) from material collected between 1985 and 2011. Complementary to this we included in situ and SEM pictures of 71 taxa into the SBP. This study includes samples from almost all island groups in the TWA (Fig. 1) from depths ranging between 2 and $950 \mathrm{~m}$, covering different geomorphological zonations as described in Reed and Pomponi (1997). The phylogenetic affinities of 31 out of the 35 'lithistid' Tetractinellida genera are reconstructed. Furthermore, our results reveal a new clade including all rhizoclone desma bearing lithistids plus Stupendidae and Thrombidae, and document potential new genera/species and occurrences for the TWA. With this systematic groundwork the molecular phylodiversity of eight island regions in the TWA and the relative abundance and bathymetric distribution patterns of 218 samples representing nine desma-bearing families are analysed and discussed suggesting 
that the present-day 'lithistid' fauna is as diverse as the fauna from the Mesozoic.

\section{MATERIALS AND METHODS}

\section{Specimen collection and identification}

Between 1995 and 2011, sponge samples were collected from the Tropical Western Atlantic (TWA) by the Johnson-Sea-Link (JSL) I and II submersibles operated by the HBOI, Fort Pierce, Florida and by scuba diving during several expeditions to the Bahamas, Cuba, Florida Keys, Curaçao, Turks and Caicos, Puerto Rico, Honduras, Jamaica, Guadeloupe, Gulf of Mexico and Bonaire (Fig. 1). These expeditions aimed to conduct a biodiversity inventory and collect samples for biomedical research focused particularly on sponges, octocorals and algae. Various habitats from the fore reef slopes and escarpments to the deep shelf slopes were sampled using either a claw, suction tube or scoop in depths from 0-1000 m. Sponge samples from this collection, were pre-identified by S.P. and M.K., and frozen and/or stored in $70 \%$ ethanol. For comparison, additional material from the Southwest Pacific (New Caledonia and New Zealand), and Indo-Pacific region, in the National Institute of Water and Atmospheric Research (NIWA) collection in Auckland and its invertebrate collection (NIC) in Wellington, New Zealand, were subsampled for molecular investigations. This material included subsamples of tetractinellids, which were collected by scuba diving during several expeditions across the Indo-Pacific and New Zealand, led by the Coral Reef Research Foundation (CRRF) in Republic of Palau, identified by M.K. Six specimens (three Geodia spp. and three Cinachyrella spp.) from Jamaica and Norway were added from the Bavarian State Collection of Zoology (ZSM) in Munich, Germany (identification by Helmut Lehnert). Detailed information for all novel samples sequenced is provided in the Supplementary Material.

Undetermined samples from the TWA (all HBOI subsamples) were identified to the genus level according to their phylogenetic position relative to known species. Based on this, we selected 71 samples with distinct genotypes for a deeper morphological investigation. For those taxa we examined deck pictures, and prepared thick sections as well as spicule and skeleton stubs for scanning electron microscopy (SEM). We used the methodology outlined in Pisera and Pomponi (2015) to illustrate and evaluate morphological characters. Based on this, 249 specimens could be identified to genus and/or species level. Morphological documentation for the 71 representative specimens are provided in the Sponge Barcoding Project (SBP) (http://www.spongebarcoding.org/). SEM stubs and spicule slides including thick sections are deposited at the Bavarian State Collection for Paleontology and Geology (BSPG) Munich, Germany under accession numbers XXXX.

\section{Molecular investigations}

Genomic DNA was isolated from small pieces of sponge tissue preserved in $70 \%$ ethanol using a modified protocol of the DNeasy (Qiagen) Blood and Tissue Kit, which included an additional centrifugation step just before transferring the lysate to the spin column. A Nano-Drop 1000 Spectrophotometer (Thermo Scientific) was used to quantify the isolated genomic DNA. Amplification of a fragment of the mitochondrial cytochrome c oxidase subunit 1 (coxl, partial $\approx 659 \mathrm{bp}$ ) was performed using the primers dgLCO1490 and dgHCO2198 (Meyer, Geller and Paulay, 2005). Additionally, a fragment of an unlinked nuclear ribosomal gene (28S; partition C1-D2, 768-832 bp) was amplified using the forward C1'ASTR (Cárdenas et al., 2010) and the reverse universal D2 (Lê, Lecointre and Perasso, 1993) primers. Both amplifications follow the PCR protocol and settings outlined in Schuster et al. (2015). Amplification success was checked on a $1.5 \%$ agarose gel. For the majority of the $28 \mathrm{~S}$ fragments we observed an additional non-specific shorter band at $\approx 650 \mathrm{bp}$, which was subsequently identified as originating from a bacterial template. Therefore, separation of double bands and PCR clean-up was performed 
using a modified freeze-squeeze method (Tautz and Renz, 1983), as described in Schuster et al (2015). For sequencing of the $28 \mathrm{~S}$ fragment, $6 \mu \mathrm{l}$ of the remaining supernatant from the clean-up was used with the PCR primers and BigDye Terminator v3.1 (Applied Biosystems, Forster City, CA, USA) chemicals. For sequencing of coxl we used a 1:10 dilution of the PCR products together with the PCR primers and BigDye Terminator v3.1 chemicals. Sequencing was carried out on an ABI 3730 Genetic Analyzer at the Sequencing Service of the Department of Biology (LMU München). Sponge origin of novel sequences were tested by BLAST searches against NCBI GenBank (https://blast.ncbi.nlm.nih.gov/Blast.cgi). Raw trace files were post- processed

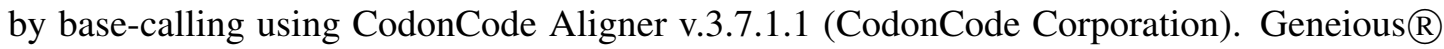
v.8.1.8 (http://www.geneious.com, Kearse et al., 2012) was used for the assembly of forward and reverse reads. Sequences will be deposited at the European Nucleotide Archive (ENA) and the SBP under accession numbers \#1794 to \#2108.

\section{Phylogenetic reconstructions}

Alignments were generated separately for cox 1 and 28S using MAFFT v.7 under the L-INS-I algorithm (Katoh and Standley, 2013) because of heterogeneous taxon sampling and moderate sequencing success of coxl. Saturation of both markers was evaluated using Xia's test (Xia et al., 2013) as implemented in DAMBE v5.1.5 (Xia, 2013) which compares an estimated substitution saturation index (Iss) to a critical substitution saturation index (Iss.c). For the coxl dataset, sequences of Halichondria panicea (Pallas, 1766) (subclass: Heteroscleromorpha Cárdenas, Pérez and Boury-Esnault, 2012, order Suberitida Schmidt, 1870) and Aplysina aerophoba (Nardo, 1833) (subclass Verongimorpha Erpenbeck et al., 2012, order Verongiida Bergquist, 1978) were chosen as outgroups. For the $28 \mathrm{~S}$ dataset sequences of the order Sphaerocladina were chosen as outgroup. All outgroups have been used in earlier phylogenetic studies on tetractinellids (see e.g. Schuster et al., 2015; Kelly and Cárdenas, 2016). The final coxl alignment comprised 307 sequences of which 122 are newly generated sequences for this study. The alignment was 635 bp long, of which $295 \mathrm{bp}$ were constant, $40 \mathrm{bp}$ were parsimony uninformative and $300 \mathrm{bp}$ were parsimony informative. The final $28 \mathrm{~S}$ alignment comprised 474 sequences of which 305 are newly generated sequences for this study. In total this alignment was $905 \mathrm{bp}$ long, of which 325 bp were constant, 66 bp parsimony uninformative and 514 bp parsimony informative. Both alignments from this study are freely available at OpenDataLMU doi.:XXXXXX. Phylogenetic tree reconstructions for both datasets were performed on a parallel version of MrBayes v3.2.4 (Ronquist et al., 2012) on a Linux cluster. The most generalized GTR+G+I evolutionary model, indicated as the most suitable by jModelTest v.2.1.7 (Darriba et al., 2012), was used. Analyses were run in two concurrent runs of four Metropolis-coupled Markov-chains (MCMCMC) for $100,000,000$ generations and stopped when the average standard deviation of split frequencies dropped below 0.01 . The first $20 \%$ of the sampled trees were removed as Burn-in from further analyses.

\section{Inclusive molecular phylodiversity and abundance analyses}

The Inclusive Phylogenetic Diversity $\left(\mathrm{PD}_{\mathrm{I}}\right)$ is the sum of all branch lengths of a gene tree connecting a set of taxa from the root of the tree to the tips of all phylogenetic branches spanned by this set of taxa (see e.g. Lewis and Lewis, 2005). To evaluate the $\mathrm{PD}_{\mathrm{I}}$, a Maximum Likelihood (ML) tree was first calculated from the most comprehensive dataset (28S, C1-D2 partition) using RAxML 7.2.8 (Stamatakis, 2014). The GTRGAMMA nucleotide evolutionary model selected by jModelTest v.2.1.7 (Darriba et al., 2012) was taken with 1000 fast pseudo-replicated bootstraps. The resulting tree topology was used to calculate the PDI for several areas in the TWA using a modified python script from Vargas et al. (2015). All non-TWA genera and all TWA genera less than five were excluded from this analysis. In total, the $\mathrm{PD}_{\mathrm{I}}$ of Bonaire, Curaçao, Florida, 
Honduras, Jamaica, Puerto Rico and Turks and Caicos was calculated. In order to compensate for different sampling efforts across the seven regions, rarefaction curves (Sanders, 1968) were used for each location. The rarefaction curves were generated in RStudio (RStudio Team, 2014). Both scripts are available at https://bitbucket.org/molpalmuc/.

The relative abundance of eight 'lithistid' families from five depth zones $(0-60 \mathrm{~m}$; 61-150 $\mathrm{m} ; 151-300 \mathrm{~m} ; 301-600 \mathrm{~m} ; 601-914 \mathrm{~m}$ ) from the TWA was plotted and illustrated using ggplot2 (Wickham, 2009) as implemented in RStudio. These depth zonations follow Reed and Pomponi (1997) and Pomponi et al. (2001), which are based on the geomorphological observations of the sites sampled.

\section{RESULTS AND DISCUSSION}

\section{Integrative morphological and molecular systematics of 'lithistid' demosponges with focus on TWA species Higher-taxa relationships of desma-bearing tetractinellids}

The 296 lithistid sequences of at least 88 species from 27 genera ( 35 known) constitute the largest and most comprehensive taxon set on desma-bearing tetractinellids to date. Our phylogenies (Fig. 2) corroborate the monophyly of Tetractinellida, currently including the suborders Astrophorina and Spirophorina (Morrow and Cárdenas, 2015) (Fig. 2C). In addition, the affinity of eight desma-bearing families to the suborder Astrophorina (Cárdenas et al., 2011; Morrow and Cárdenas, 2015; Schuster et al., 2015) is confirmed (Fig. 2A-C). The 28S phylogeny (Fig. 2A) indicates a sister relationship of Astrophorina and Spirophorina. In both gene trees (Fig. 2A, B) desma-bearing tetractinellids do not group with the Spirophorina (only represented by the Tetillidae in our sampling). In both gene trees the rhizomorine-bearing families Scleritodermidae, Siphonidiidae and Azoricidae form a clade (Fig. 2). However, Gastrophanella (Siphonidiidae) is distinct and sister (1.0 Posterior Probability (PP)) to Scleritodermidae/Siphonidiidae/Azoricidae in the 28S phylogeny (Fig. 2A). This sister-group relationship could not be corroborated by coxI analysis as no sequence of Gastrophanella could be generated. We suspected an intron insertion within cox 1 due to the discovery of these in closely related rhizomorine-bearing genera (Setidium Schmidt, 1879, Microscleroderma Kirkpatrick, 1903, Aciculites Schmidt, 1879, Scleritoderma Sollas, 1888) (Schuster et al., 2017). Based on this, various primer sets suggested by Schuster et al. (2017) were tested, however, without success. We suspect that Gastrophanella has one or several intron insertions in the coxl gene in a yet unknown position. By including several additional rhizomorine-bearing genera such as Gastrophanella, Leiodermatium, Siphonidium and Amphibleptula in our datasets, the family Thrombidae could not be recovered within Astrophorina as hypothesized by the Systema Porifera (Hooper and Van Soest, 2002). The 28S gene tree recovers Thrombidae as sister to all rhizomorine-bearing tetractinellids, but this relationship is not supported (0.72 PP) (Fig. 2 and Fig. 3) and needs further investigation, including also 28S for Stupendidae Kelly and Cárdenas 2016, a recently established new family (Kelly and Cárdenas, 2016). In the coxl phylogeny (Fig. 2B) Stupendidae is included and a highly supported sister taxon to Scleritodermidae/Siphonidiidae/Azoricidae. It should be noted that Thrombidae and Gastrophanella are missing in the coxl phylogeny (Fig. 2B).

\section{Intra-subordinal relationships of astrophorine 'lithistids'}

The majority (15 out of 23) of the currently known tetractinellid families are located within the Astrophorina (Morrow and Cárdenas, 2015). This includes eight desma-bearing families (Corallistidae, Isoraphiniidae Schrammen, 1924, Macandrewiidae Schrammen, 1924, Neopeltidae, Pleromidae, Phymaraphiniidae Schrammen, 1924, Phymatellidae and Theonellidae) and seven non-desma bearing families (Cárdenas et al., 2011; Morrow and Cárdenas, 2015; Schuster et al., 2015) (see also Fig. 2A-C). Thus, the present study supports earlier findings, which were 
based on lower taxon sampling and additionally provides deeper insights into the intraspecific relationship of desma-bearing astrophorids.

The family Theonellidae consists of the genera Discodermia du Bocage, 1869, Manihinea Pulitzer, Finali, 1993, Racodiscula Zittel, 1878, Siliquariaspongia Hoshino, 1981 and Theonella Gray, 1868. Theonellidae possesses tetraclone desmas and phyllotriaenes to discotriaenes as characteristic megascleres. Typical microscleres are acanthorhabds, spirasters and amphiasters (Pisera and Lévi, 2002a). Until now, only Theonella and Discodermia species as well as one Manihinea sp. were sequenced in different phylogenetic studies using $18 \mathrm{~S}, 28 \mathrm{~S}$ and cox 1 (see e.g. Redmond et al., 2013; Hall, Ekins and Hooper, 2014; Schuster et al., 2015). By providing sequences for all known genera, our 28S phylogeny (Fig. 3) recovers Theonellidae as monophyletic $(\mathrm{PP}=1.0)$, thus conclusively support earlier findings of Schuster et al. (2015), while the coxl phylogeny (Fig. 4) lacks support in this respect. The 28S phylogeny indicates the monophyly of the genera Discodermia, Manihinea, Racodiscula and a potential new taxon, here denoted as Theonellidae sp., a potential new genus mainly distinct by the layered network of tetracone desma with smooth rays and strongly tuberculated tips and the less abundant microscleres on the ectosome (SBD2102-2106). The sister relationship of Manihinea conferta to Theonella sp. 1 is highly supported ( $\mathrm{PP}=0.99$ ) by $28 \mathrm{~S}$ (Fig. 3), whereas it is not supported by cox1 (Fig. 4). A close relationship of Theonella and Manihinea was observed in an earlier study by Redmond et al. (2013) using a nearly complete $18 \mathrm{~S}$ gene fragment, but unsupported. The genus Discodermia is sister to a clade consisting of Manihinea+Theonella+Siliquariaspongia, which is sister to Racodiscula+Theonellidae sp.

The genus Racodiscula is highly supported $(\mathrm{PP}=1.0)$ as sister to Theonellidae sp. Although the outer morphology of Theonellidae sp. (SBD2106 A-D) is very similar to that of Racodiscula, it differs in spicule composition, desma and skeleton structure: the usually abundant spinose microacanthorhabds, covering the surface of Racodiscula species (SBD2065) building a dense crust on the surface, are rarer or even absent in Theonellidae sp. Instead of microaconthorabds, phyllo- to discotriaenes are the main components of the dense surface crust. In addition, Theonellidae sp. possesses desmas with smooth rays and strongly tuberculated tips (SBD2105 and SBD2106) building a layered network (SBD2102), which clearly differs from Racodiscula (Schuster et al. 2018).

Theonella mirabilis (de Laubenfels, 1954) was first named within the homoscleromorphid genus Placinalopha (Class Homoscleromorpha, Order Homosclerophorida, Family Plakinidae) on the possession of what de Laubenfels described as 'lophotetractines'. The key size and shape differences between the 'tetralophs' of T. mirabilis and other Placinalopha species were noted by Muricy and Díaz (2002), who suggested that the species mirabilis had a more likely affinity with species in family Theonellidae. 28S sequences (Fig. 3) unite specimens identified as Theonella mirabilis in a single clade with a specimen identified as T. conica (Kieschnick, 1896) which also has tetraloph-like desmas, suggesting that species with non-articulated 'tetraloph' desmas may be monophyletic and separate from other Theonella spp. However, coxl sequences (Fig. 4) separate T. mirabilis into two groups, nesting them within diverse species of Theonella. Theonella mirabilis is very similar in spicule complement to the type species of the genus Siliquariaspongia, S. japonica Hoshino, 1981 (family Theonellidae), although the latter lacks the strongyles and possesses frilly discotrianes, the latter occasionally recorded in T. mirabilis. Our phylogenies clearly place all of the sequenced Theonella mirabilis species within the Theonella+Manihinea clade (Fig. 3), confirming that this species belongs to the family Theonellidae. This result is supported by the discovery of potent new depsipeptides mirabamides A-D, that inhibit HIV-1 infection, adding to a small class first exemplified by the papuamides from various Theonella spp. (Plaza et al. 2007).

The family Macandrewiidae is monogeneric with currently seven valid species (Van Soest et 
al. 2018a). Until know, only Macandrewia rigida Lévi and Lévi, 1989 from the Solomon Islands has been sequenced (28S C1-D2 region, LN624160, G317931) (Schuster et al., 2015). The present study includes a further sequence of an undescribed Macandrewia sp. from the Bahamas (909 m depth), which clearly differs from M. rigida (Fig. 3). Morphological differences in desmas (SBD2004) corroborate the genetic difference to $M$. rigida and provide further evidence of a possible new species, which would be the first record in the TWA. Nevertheless, further morphological observations and comparison with the type material of $M$. rigida as well as its sequences are needed to conclusively describe and distinguish this potential new species. Both Macandrewia species group within the Geodiidae, close to the Erylinae Sollas, 1888, within a clade of non-desma bearing astrophorins (Calthropella Sollas, 1888, Caminella Lendenfeld, 1894) (Fig. 3). This relationship is currently not supported by morphology (Cárdenas et al., 2018) and in any case suggests a distinct evolutionary history of Macandrewia to other 'lithistid' families Corallistidae and Neopeltidae (Schuster et al., 2015), where Macandrewia was previously allocated (Kelly, 2000; Pomponi et al., 2001).

The family Phymatellidae currently includes three genera: Neoaulaxinia, Neosiphonia and Reidispongia. The present comprehensive study does not recover Phymatellidae as monophyletic and is therefore in contrast to Schuster et al. (2015). Instead, a highly supported sister group relationship of Neoaulaxinia Pisera and Lévi, 2002 to the neopeltid genus Daedalopelta Sollas, 1888 is observed and Reidispongia coerulea Lévi and Lévi, 1988 is sister to this clade (Fig. 3). This relationship is in conflict with morphological hypotheses as Phymatellidae is characterized by ectosomal dichotriaenes, while Neopeltidae has ectosomal pseudophyllotriaenes or pseudodiscotriaenes (Pisera, 2002). Until now only one species of Daedalopelta is known (D. nodosa Sollas, 1888 from Florida, Gulf of Mexico and Southern Caribbean), which clearly differs from our Daedalopelta specimens. Therefore, morphological characters of both sequenced Daedalopelta species were further investigated and illustrated (SBD1894). Both species are potentially new to science. Daedalopelta sp. 1 (SBD1894) possesses the characteristic pseudophyllotriaenes (SBD1894) known from the Neopeltidae, while Daedalopelta sp. 2 (SBD1895) possesses dichotriaenes characteristic for the Phymatellidae. These dichotriaenes, however, have a unique shape with indented cladomes (SBD1895). A spicule drawing of Corallistes tubulatus Van Soest and Stentoft, 1988 from Barbados, now Neophrissospongia tubulata, resembles those unique dichotriaenes implying that $N$. tubulata may need to be reallocated to Daedalopelta. Interestingly, dichotriaenes of Daedalopelta sp. 2 resemble those of the fossil Gignouxia niciensis Moret, 1926 (Corallistidae) from the Late Cretaceous (Pl. XVIII, Fig. 2, 2' fig-txt 37). We suggest to allocate Gignouxia Moret, 1926 to the family Phymatellidae. Gignouxia will include Daedalopelta sp. 2 as well as Daedalopelta tubulata comb. nov.

Neopeltidae polyphyly is given by the highly supported $(\mathrm{PP}=1.0)$ sister relationship of the newly sequenced species Neopelta sp. to the non-desma bearing astrophorid Neamphius huxley de Laubenfels, 1953 (Fig. 3). Morphologically, these two species only share choanosomal amphiasters with spiny rays and microxeas. Thus, monocrepid desmas and pseudodiscotriaenes characterizing Neopelta were lost in Neamphius. Spicule losses and gains are not uncommon within tetractinellids and have frequently been shown (Chombard, Boury-Esnault and Tillier, 1998; Cárdenas et al., 2011; Schuster et al., 2015). Nevertheless, these two genera form a robust sister clade to a non-desma bearing Ancorinidae clade consisting of Stelletta Schmidt, 1862, Ancorina Schmidt, 1862, Asteropus Sollas, 1888 and Stryphnus Sollas, 1886.

Corallistidae is another major family dominating the HBOI collection and subsequently our phylogenies (Fig. 3 and 4). These taxa were the focus of biomedical investigations (e.g. Haar et al., 1996; Wright, 2010), thus targeted during the HBOI expeditions. Therefore, whether the genera Discoderma and Corallistes Schmidt, 1870 are over-represented in this study due to their increased sampling, or whether they actually dominate the sponge fauna in the TWA regions 
remain obscure and awaits further investigations. Even though Corallistes were frequently sampled in the past and 15 species are described to date (Van Soest et al. 2018b), only five sequences are published (Kelly Borges and Pomponi, 1994; Chombard, Boury-Esnault and Tillier, 1998; McInerney, Adams and Kelly, 1999). With 52 Corallistes specimens sequenced, this study presents the largest data set to data and reveals the monophyly of this genus (28S, $\mathrm{PP}=1.0$, Fig. 3). Corallistes typus Schmidt, 1870 specimens, type species of the genus, were examined (SBD1888, 1889, 1892) and sequenced (Fig. 3 and 4). In addition, a Corallistes isabela Desqueyroux-Faúndez and Van Soest, 1997 sample from Honduras was sequenced. Until this study Corallistes isabela was only known from the Eastern Pacific (Galápagos) and discussed as endemic to the Galápagos (Desqueyroux-Faúndez and Van Soest, 1997 and Schuster et al., 2018). In addition, six Corallistes (C. sp. 2 to $C$. sp. 7, see Fig. 3) differ by 1-3 bp in the $28 \mathrm{~S}$ fragment, while no differences were found in coxl. Morphological differences are observed between $C$. sp. 2 (SBD1872) and C. sp. 4 (SBD1879). For example, $C$. sp. 2 has long (700 $\mu \mathrm{m}$ ) thin ectosomal oxeas (SBD1872 A), while $C$. sp. 4 has ectosomal styles (SBD1879 D) and subectosomal microxea with spined surfaces (SBD1879 C). Morphological identifications are in progress and necessary to discriminate the remaining Corallistes species.

The polyphyletic corallistid genus Herengeria Lévi and Lévi, 1983, only known from the Pacific Norfolk Ridge, New Caledonia, and New Zealand (Schlacher-Hoenlinger, Pisera and Hooper, 2005; Kelly et al., 2009), was sequenced (Fig. 3) and morphologically illustrated (SBD1949) in the present study for the first time from the Bahamas and Honduras, representing a new genus for the Atlantic. Only two species of Herengeria are described (H. auriculata Lévi and Lévi 1988 and H. vasiformis Schlacher-Hoenlinger, Pisera and Hooper, 2005). The morphological observations delimit the new TWA species from $H$. auriculata (Schlacher-Hoenlinger and Hooper, 2005) due to a lack of subectosomal rhabd-like spirasters. With respect to molecular markers, Herengeria spp. from the TWA are distinct from $H$. vasiformis/Herengeria sp. from the Pacific (Fig. 3). Our 28S (Fig. 3) and coxl (Fig. 4) phylogenies strongly support Neoschrammeniella Pisera and Lévi, 2002 as sister to the Herengeria/Isabella clade. Currently, seven valid species of Neophrissospongia Pisera and Lévi, 2002 are described (Van Soest et al. 2018c). Only a few Neophrissospongia sequences (two 28S and one 18S) from the Pacific Ocean are currently published. However, in order to gain a better understanding of their geographical distribution and genetic differences, additional material from the Caribbean were sequenced in this study: Their resulting 28S phylogeny clearly separates Neophrissospongia from the Pacific and the Caribbean Islands. Neophrissospongia sp. 1 from different Caribbean Islands is sister ( $\mathrm{PP}=1.0)$ to N. microstylifera and Neophrissospongia sp. 3, from the Pacific (Fig. 3). A further Caribbean Neophrissospongia species (sp. 2) forms a robust sister clade to Neophrissospongia from the Eastern Pacific (Galápagos, Panama) (Fig. 3).

A clade of six as yet unidentified specimens from the HBOI collection (SBD1814) is sister to the monophyletic Neophrissospongia ( $\mathrm{PP}=1.0$, Fig. 3). We assume that this clade consists of species from the as yet unsequenced genus Awhiowhio Kelly, 2007 from the Pacific based on morphological evidence. These show similar mega- and microsclere types to Awhiowhio such as dicranoclone desmas and smooth dichotriaenes in Awhiowhio sp. 1 (SBD1815), most similar to the Awhiowhio osheai from New Zealand (Kelly, 2007), but slightly different in terms of desma ornamentation. Streptaster microscleres and acanthose microrhabds in Awhiowhio sp. 1 (SBD1814, 1815) differ from those in Awhiowhio osheai in sizes and shapes (SBD1814). The coxl phylogeny (Fig. 4) indicates the sister group relationship of Awhiowhio osheai Kelly, 2007 to Neophrissospongia. A close relationship of Awhiowhio to Herengeria as suggested by Kelly (2007) based on morphological features is not supported by any of our phylogenies. Instead, both markers independently suggest a close relationship (strongly supported by $\mathrm{PP}=1.0$ ) to Neophrissospongia. The genus Pleroma Sollas, 1888 (family Pleromidae) is recovered as 
paraphyletic in both phylogenies (Fig. 3 and 4). Pleroma menoui Sollas, 1888 is distant to other Pleroma spp. (including the type species $P$. turbinatum) in a close relationship to Corallistidae (Fig. 3 and 4).

\section{Intra-subordinal relationships of spirophorine 'lithistids'}

The suborder Spirophorina is characterized by sigmaspire microscleres and its members share triaene spicules with Astrophorina. Currently, three families are known: Samidae Sollas, 1888, Spirasigmidae Hallmann, 1912 and Tetillidae Sollas, 1886, whereas the latter is the largest in terms of genera and species (e.g. Van Soest and Hooper, 2002). The relationships of major clades within our coxl and 28S phylogenies (Fig. 3 and 4) were in concordance with the findings of Carella et al. (2016) and Schuster et al. (2017). The latest revised classification of Morrow and Cárdenas (2015) included the desma-bearing families Azoricidae, Scleritodermidae and Siphonidiidae within Spirophorina. Since then, several studies (Schuster et al., 2015, 2017; Kelly and Cárdenas, 2016) using coxl, 18S and 28S markers showed the separation of all rhizomorinebearing sponges from Spirophorina (=Tetillidae) The present enlarged dataset corroborates again the absence of desma-bearing sponges in Spirophorina and their grouping in a well supported clade along with the Stupendidae and the Thrombidae (Fig.2). In order to establish this clade as a new taxa, we await further molecular data from the latter two families (work in progress, MK and PC).

\section{Subordinal structure of Tetractinellida}

Kelly and Cárdenas (2016) provided strong support for families Azoricidae, Scleritodermidae and Siphonidiidae within a new suborder, supported in part by the common possession of rhizomorine desmas.

Regarding Azoricidae, Maldonado et al. (2015) discovered a dense and large aggregation of Leiodermatium pfeifferae Carter, 1873 on seamounts in the Mediterranean building complex reef-forming structures. Even though Leiodermatium Schmidt, 1870 has very few diagnostic characters (no microscleres) to discriminate between species (Pisera, 2002; Pisera and Lévi, 2002b), 11 species are valid to date (WPD access Aug. 2017). In the present study we sequenced the 28S C1-D2 fragment for 52 Leiodermatium specimens from several regions in the TWA (Fig. 3) representing at least 8 species and the largest sequenced dataset for this genus to date. The monophyly of Leiodermatium is highly supported by our $28 \mathrm{~S}$ phylogeny ( $\mathrm{PP}=1.0$; Fig. 3). The amplification of coxl unfortunately failed, most likely due to the presence of introns similar to other rhizomorine-bearing genera like e.g. Microscleroderma and Scleritoderma (Schuster et al., 2017). Preliminary morphological investigations (SBD1966,1967,1959,1965,1956,1955,1975,1977,1976,1994,2000,2001,2003) adumbrate detailed differences of Leiodermatium spp., in particular their surfaces (oscules and ostia sizes), diactines and desma morphology. For instance Leiodermatium sp. 1 (SBD1955) has large and marginate oscules, while Leiodermatium sp. 6 (SBD1994) and Leiodermatium sp. 8 (SBD2003) have large but elevated oscules on exterior margins, in contrast to Leiodermatium sp. 7, whose oscules are small and closely distributed. Based on molecular and morphological data we propose eight different species of Leiodermatium (Fig. 3) in the TWA, however further morphological investigations are needed to corroborate this assumption. Leiodermatium is unsupported ( $\mathrm{PP}=0.65)$ sister to a clade of Siphonidium spp. (Siphonidiidae); the same relationship was revealed with small fragment of the $18 \mathrm{~S}$ gene (482 bp) for Leiodermatium sp. (Kelly-Borges and Pomponi, 1994, Kelly and Cárdenas, 2016). Further investigation and a review of all extant and fossil Leiodermatium species is suggested to better understand the geographical distribution and recent diversification of this paleontological important group.

Within the polyphyletic rhizomorine family Scleritodermidae, its genera Aciculites is polyand Scleritoderma is paraphyletic, while Microscleroderma, Amphibleptula Schmidt, 1879 and 
Setidium Schmidt, 1879 are monophyletic (Fig. 3). The genus Amphibleptula is currently monospecific with A. madrepora Schmidt, 1879 from the Caribbean (Pisera and Lévi, 2002c). Morphologically, A. madrepora is very similar and easy to confuse with Microscleroderma spirophora Lévi, 1960 as discussed in Van Soest and Stentoft (1988). Amphibleptula is here sequenced for the first time and our 28S phylogeny shows Microscleroderma and Amphibleptula sp. 1 as sister groups, although unsupported in the $28 \mathrm{~S}$ phylogeny $(\mathrm{PP}=0.63)$. Morphological observations (SBD1802,1803) provide conclusive evidence that our three samples are Amphibleptula species, due to their dense tuberculated/blunt spinose rhizoclones, the protruding bundles of oxeas in the oscula area (SBD1802,1803) as well as the presence of sigmaspires (SBD1802). Differences to A. madrepora are the diactine spicules present in all three Amphibleptula sp.1. In addition, fusiform spined microxeas and acanthorhabds are found in the specimen from Jamaica (HBOI 1-IX-93-1-006, SBD1802). To conclude, we sequenced two potential new species of Amphibleptula with clear unique morphological characters, different from A. madrepora.

During this study a new suborder Thoosina was suggested by Carballo et al. 2018 including the genera Thoosa and Alectona so far. However, since no desma-bearing tetractinellids are grouping within this suborder, we decided to not further discuss Thoosina in the scope of this study.

\section{Molecular phylodiversity of TWA desma-bearing demosponges}

In the present study the inclusive phylodiversity was calculated for Bonaire, Curaçao, Florida, Honduras, Jamaica, Puerto Rico, Turks and Caicos and the Bahamas (Fig. 5). The PDI analyses disclosed a high variation within the TWA locations (Fig. 5). At comparable sampling efforts, the highest $\mathrm{PD}_{\mathrm{I}}$ was observed in Jamaica (Fig. 5A) indicating a high biodiversity in this region, closely followed by the Bahamas (Fig. 5B). At sample size of 20, Curaçao and Florida showed the lowest $\mathrm{PD}_{\mathrm{I}}$, followed by Turks and Caicos and Honduras. The high $\mathrm{PD}_{\mathrm{I}}$ of 'lithistid' demosponges calculated for the Bahamas is in agreement with the findings of Reed and Pomponi (1997), and may be explained by the high habitat diversity observed in this region (Reed and Pomponi, 1997) and their close proximity to the species-rich Atlantic (see e.g. Carvalho, Pomponi and Xavier, 2015). Even though Turks and Caicos are close to the Bahamas and the Atlantic, a much lower $\mathrm{PD}_{\mathrm{I}}$ was calculated, maybe due to less habitat diversity.

\section{Bathymetric distribution and relative abundance of TWA desma-bearing families}

The evaluation of the relative abundance of eight 'lithistid' families within each depth zone is based upon 234 specimens collected from eight localities in the TWA (Fig. 6). Theonellidae and Corallistidae are the two dominant families in the present dataset, and assumed to be the dominant families in the TWA (Pomponi et al., 2001). While Theonellidae dominate depth zones of 0-151 m, Corallistidae are more abundant in depth zones of 151-600 m. This corroborates the result of Pomponi et al. (2001) showing that Discodermia (Theonellidae) is the dominant genus from 0-151 m, while Corallistes (Corallistidae) dominates the zone of 151-914 m. An explanation for this might be that Corallistidae have a dense rigid skeleton of dicranoclone desmas, while Theonellidae possess a less articulated skeleton of tetraclone desmas. Thus, it can be hypothesized, that 'lithistids' with a hyper-silicified dense desma skeleton like Corallistidae occur in deeper zones $\geq 300 \mathrm{~m}$, while those with a less dense desma skeleton like most of the Theonella species (Theonellidae) moved into more shallow water habitats, as less silica is required for their skeleton construction. This trend was also observed in the South West Pacific (Kelly et al., 2007; Hall, Ekins and Hooper, 2014). As Corallistidae and Theonellidae are considered to be polymorphic (Pisera and Lévi, 2002a,d), it is difficult, to draw any conclusion of different depth zones or habitats influencing growth form patterns in these two families.

However, further 'lithistid' families with a similar bathymetric trend are observed and growth 
forms are suggested to play a role in the bathymetric distributions of 'lithistids'. For instance Leiodermatium spp. (Azoricidae) are abundant (27 specimens) in depth zones 301-1000 m. Similar to Corallistidae Leiodermatium possess a dense heavily articulated skeleton, but of strongly spinose rhizoclone desmas. The growth form of Leiodermatium species are described as being foliated or vase to ear-shaped (Pisera and Lévi, 2002b). Such growth forms are suggested to improve the water circulation in sponges, in particular of those in the deep-sea habitats, and to be more resistant to higher water viscosity and scarcity of particles (Levinton, 1982; Gage and Tyler, 1991). Many vase to cup or ear-shaped sponges have their inhalant pores facing the outer side and exhalant openings on the upper side separating incoming and processed water (Sará and Vacelet, 1973), which may reduce any negative effect on filtering due to a sedimentation. This is in contrast to Siphonidiidae, a family represented in this analysis by the genera Gastrophanella and Siphonidium, which are rather encrusting or irregular cylindrical, thus more abundant in the depth zone of $61-150 \mathrm{~m}$.

Scleritodermidae occurred more often on vertical walls in depth $301-600 \mathrm{~m}$, but was also not observed to be a major component of the 'lithistid' fauna in the TWA. The greatest number of desma-bearing demosponges were found in depth zone 301-600 m (87 specimens), with Corallistidae as the dominant family (34 specimens) followed by Azoricidae with 27 specimens. Diverse habitats from fine mud and sand slopes to rock pinnacles, boulders and vertical walls in this depth zone (Fig. 6) could be a possible explanation. The families Neopeltidae and Macandrewiidae are rare in our study with only one species discovered at $909 \mathrm{~m}$ depth on a vertical wall in the Bahamas (Macandrewia sp.), and two Daedalopelta sp. species collected from the Bahamas at 301-600 m. This corroborates the findings of Pomponi et al. (2001), because they found one species of Daedalopelta nodosa at $452 \mathrm{~m}$ in the Bahamas, one Neopelta perfecta in $116 \mathrm{~m}$ depth from Grenada and one Macandrewia clavatella in the southwest coast of Florida. These families and species are also found to be rare in the Southwest Pacific (Lévi, 1991; Kelly, 2000). Besides the tetractinellid 'lithistid' sponges, we noted that other desma-bearing sponge lineages, such as family Vetulinidae (Order Sphaerocladina) constitute only a minor component in any depth-zone in the TWA.

Further testing is required to assess whether geomorphological conditions resulting of a variety of complex tectonic interactions (e.g., strike-slip faults, thrust fault, subduction and seafloor spreading in Cayman Trough, see Fig. 1), directly affect diversity and bathymetric distribution of 'lithistids' in the TWA (Fig. 6).

\section{CONCLUSION}

In summary, this is the first integrative approach using molecular and morphological data on TWA 'lithistid' demosponges, thus contributing to a better understanding of their phylogenetic affinities, diversity and bathymetric distribution patterns. The present study points to specimens/groups in need of deeper taxonomic investigations and revision, however, additional morphological as well as other independent markers are needed. With recent evidence (Pomponi et al. 2001) that 'lithistids' are dominant components among all investigated TWA regions, we suggest a comparable diversity to the Pacific 'lithistids' as well as to the Mesozoic fauna. Furthermore, there is a clear shift of lithistids with a rigid and heavily articulated desma towards deeper habitats (Corallistidae and Azoricidae), whereas 'lithistids' with a less articulated skeleton tend to occur in more shallow habitats (Theonellidae and Siphoniidae). A major effect causing this shift is the availability of silica in the ocean throughout time. Our robust phylogeny enables relaxed molecular clock analyses in conjunction with the rich fossil record of lithistids to better correlate such shifts to geological/geochemical events in the past. 


\section{ACKNOWLEDGMENTS}

We acknowledge the Government of the Bahamas, Curaçao, Jamaica, Turks and Caicos, Bonaire and St. Vincent Islands, Martinique, Guadeloupe, Puerto Rico, Cuba, Jamaica, Honduras, Gulf of Mexico, and the Philippines, for granting permission to conduct research in their territorial waters. Thirty-two new specimens and associated data were supplied by the NIWA Invertebrate Collection (NIC) for sequencing; we are particularly grateful to Sadie Mills for her diligent assistance with loans. A further 31 specimens and associated images and data were supplied by Lori J. Bell and the Coral Reef Research Foundation (CRRF) for sequencing; these were collected under contract to the U.S. National Cancer Institute. We thank colleagues Amy Wright, John Reed and Megan Conkling (HBOI-FAU) for their assistance with the HBOI collection of samples and associated data. AS thanks Sergio Vargas (Dept. of Earth- and Environmental Sciences, LMU Munich, Germany) for his help with the modification of the phylodiversity script. Nicole Enghuber is thanked for her help in spicule preparations and Simone Schätzle is thanked for sequencing assistance (both Dept. of Earth- and Environmental Sciences, LMU Munich, Germany).

\section{ADDITIONAL INFORMATION AND DECLARATIONS}

This document reflects only the authors' view and the Executive Agency for Small and Mediumsized Enterprises (EASME) is not responsible for any use that may be made of the information it contains.

\section{FUNDING}

Financial support for this study was provided by the German Science Foundation to DE and GW (DFG ER 611/3-1, DFG Wo869/15-1, respectively). The LMU Mentoring and the HELGE AX:Son JOHNSON STIFTELSE provided funding for AS to visit HBOI (Florida, USA) and NIWA (National Institute of Water and Atmospheric Research, Auckland and Wellington in New Zealand). Financial support for the R/V Seward Johnson/Johnson Sea Link I+II expedition was provided by HBOI. MK's participation was funded by NIWA under Coasts and Oceans Research Programme 2 Marine Biological Resources: Discovery and definition of the marine biota of New Zealand (2016/2017 and 2017/2018 SCI). PC and SP received support from the European Union's Horizon 2020 research and innovation program through the SponGES project (Grant agreement No. 679849).

\section{COMPETING INTERESTS}

The authors declare there are no competing interests.

\section{AUTHOR CONTRIBUTIONS}

Astrid Schuster and Dirk Erpenbeck conceived and designed the study. Dirk Erpenbeck and Gert Wörheide acquired funding, contributed reagents, materials and analysis tools. Astrid Schuster, Andrzej Pisera, Paco Cárdenas, Shirley A. Pomponi and Michelle Kelly identified genera/species. Shirley A. Pomponi (HBOI), Michelle Kelly (NIWA) contributed to the sampling. Astrid Schuster performed the laboratory experiments, did the phylogenetic analyses, bathymetric distribution plot, phylodiversity analysis and SEM. Astrid Schuster wrote the manuscript, prepared figures and tables. Paco Cárdenas, Dirk Erpenbeck, Michelle Kelly, Andrzej Pisera and Gert Wörheide edited versions of the manuscript. All authors reviewed drafts of the paper. 
DATA AVAILABILITY

The following information was supplied regarding data availability:

Novel sequences will be archived at the European Nucleotide Archive (ENA) under the

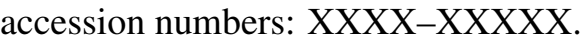

\section{REFERENCES}

Addis JS., Peterson KJ. 2005. Phylogenetic relationships of freshwater sponges (Porifera, Spongillina) inferred from analyses of $18 \mathrm{~S}$ rDNA, COI mtDNA, and ITS2 rDNA sequences. Zoologica Scripta 34:549-557.

Bergquist PR. 1970. Sponges. Hutchinson University Library, University of California Press, Berkeley and Los Angeles 1-268.

Carballo JL., Bautista-Guerrero E., Cárdenas P., Cruz-Barraza JA., Aguilar-Camacho JM. 2018. Molecular and morphological data from Thoosidae in favour of the creation of a new suborder of Tetractinellida. Systematics and Biodiversity (2018):1-10.

Cárdenas P., Rapp HT., Schander C., Tendal OS. 2010. Molecular taxonomy and phylogeny of the Geodiidae (Porifera, Demospongiae, Astrophorida) - combining phylogenetic and Linnaean classification. Zoologica Scripta 39:89-106.

Cárdenas P., Vacelet J., Chevaldonné P., Pérez T., Xavier JR. 2018. From marine caves to the deep sea, a new look at Caminella (Demospongiae, Geodiidae) in the Atlanto-Mediterranean region. Zootaxa 4466:174-196.

Cárdenas P., Xavier JR., Reveillaud J., Schander C., Rapp HT. 2011. Molecular phylogeny of the Astrophorida (Porifera, Demospongiae) reveals an unexpected high level of spicule homoplasy. PloS ONE 6:e18318.

Carella M., Agell G., Cárdenas P., Uriz M j. 2016. Phylogenetic reassessment of Antarctic Tetillidae (Demospongiae, Tetractinellida) reveals new genera and genetic similarity among morphologically distinct species. PloS ONE 11:e0160718.

Carvalho FC., Pomponi SA., Xavier JR. 2015. Lithistid sponges of the upper bathyal of Madeira, Selvagens and Canary Islands, with description of a new species of Isabella. Journal of the Marine Biological Association of the United Kingdom 95:1287-1296.

Chombard C., Boury-Esnault N., Tillier S. 1998. Reassessment of homology of morphological characters in tetractinellid sponges based on molecular data. Systematic Biology 47:351-366.

Darriba D., Taboada GL., Doallo R., Posada D. 2012. jModelTest 2: more models, new heuristics and parallel computing. Nature Methods 9:772-772.

Desqueyroux-Faúndez R., Van Soest RWM. 1997. Shallow waters Demosponges of the Galápagos Islands. Revue Suisse de Zoologie 104:379-467.

Erpenbeck D., Sutcliffe P., Cook SDC., Dietzel A., Maldonado M., Van Soest RWM., Hooper JNA., Wörheide G. 2012. Horny sponges and their affairs: On the phylogenetic relationships of Keratose sponges. Molecular Phylogenetic and Evolution 63:809-816.

Gage JD., Tyler PA. 1991. Deep-Sea Biology: A Natural History of Organisms at the Deep-Sea Floor. Cambridge: Cambridge University Press.

Giunta G., Orioli S. 2011. The Caribbean Plate Evolution: Trying to resolve a very complicated tectonic puzzle. In Sharkov EV eds. New Frontiers in Tectonic Research - General Problems, Sedimentary Basins and Island Arcs Chapter 10.

Haar ET., Kowalski RJ., Hamel E., Lin CM., Longley RE., Gunasekera SP., Rosenkranz HS., Day BW. 1996. Discodermolide, a cytotoxic marine agent that stabilizes microtubules more potently than taxol. Biochemistry 35:243-250.

Hall KA., Ekins MG., Hooper J. 2014. Two new desma-less species of Theonella Gray, 1868 (Demospongiae:Astrophorida: Theonellidae), from the Great Barrier Reef, Australia, and a 
re-evaluation of one species assigned previously to Dercitus Gray, 1867. Zootaxa 3814:451-477.

Hallmann EF. 1912. Report on the Sponges obtained by the F.I.S. 'Endeavour' on the Coasts of New South Wales, Victoria, South Australia, Queensland, and Tasmania, 1909-10. Part I. Zoological Results of the Fishing Experiments carried out by F.I.S. 'Endeavour', 11909-14.

Hoshino. 1981. Shallow-Water Demosponges of Western Japan 2. Journal of Science of the Hiroshima University (B) 29:207-289.

Katoh K., Standley DM. 2013. MAFFT Multiple sequence alignment software version 7: Improvements in performance and usability. Molecular Biology and Evolution 30:772-780.

Kearse M., Moir R., Wilson A., Stones-Havas S., Cheung M., Sturrock S., Buxton S., Cooper A., Markowitz S., Duran C., Thierer T., Ashton B., Meintjes P., Drummond A. 2012. Geneious Basic: an integrated and extendable desktop software platform for the organization and analysis of sequence data. Bioinformatics 28:1647-1649.

Kelly M. 2000. Description of a new lithistid sponge from northeastern New Zealand, and consideration of the phylogenetic affinities of families Corallistidae and Neopeltidae. Zoosystema 22:265-284.

Kelly M. 2007. The Marine Fauna of New Zealand: Porifera : Lithistid Demospongiae (rock Sponges). NIWA Biodiversity Memoir 121:1-100.

Kelly Borges M., Pomponi SA. 1994. Phylogeny and classification of lithistid sponges (Porifera: Demospongiae): a preliminary assessment using ribosomal DNA sequence comparisons. Molecular Marine Biology and Biotechnology 3:87-103.

Kelly M., Cárdenas P. 2016. An unprecedented new genus and family of Tetractinellida (Porifera, Demospongiae) from New Zealand's Colville Ridge, with a new type of mitochondrial group I intron. Zoological Journal of the Linnean Society 177:335-352.

Kelly M., Edwards AR., Wilkinson MR., Alvarez B., Cook S de C., Bergquist PR., Buckeridge S., Campbell HJ., Reiswig HM., Valentine C., Vacelet J. 2009. Phylum Porifera: sponges. In: Gordon DP ed. New Zealand inventory of biodiversity: 1 Kingdom Animalia: Radiata, Lophotrochozoa, Deuterostomia. New Zealand Inventory of Biodiversity 1, 23-46.

Kelly M., Ellwood M., Tubbs L., Buckeridge J. 2007. The lithistid Demospongiae in New Zealand waters: species composition and distribution. Porifera Research: Biodiversity, Innovation and Sustainablity 393-404.

Kieschnick O. 1896. Silicispongiae von Ternate nach den Sammlungen von Herrn Prof. Dr. W. Kükenthal. Zoologischer Anzeiger 19:526-534.

Kirkpatrick R. 1903. Descriptions of South African Sponges. Marine Investigations in South Africa 2:171-180.

Lê HLV., Lecointre G., Perasso R. 1993. A 28S rRNA-based phylogeny of the gnathostomes: first steps in the analysis of conflict and congruence with morphologically based cladograms. Molecular Phylogenetics and Evolution 2:31-51.

Lévi C. 1991. Lithistid sponges from the Norfolk Rise, Recent and Mesozoic genera. In: Reitner J, Keupp H eds. Fossil and Recent Sponges. Berlin, Heidelberg: Springer-Verlag, 72-82.

Levinton JS. 1982. Marine Ecology. New Jersey: Prentice-Hall. Lewis LA., Lewis PO. 2005. Unearthing the molecular phylodiversity of desert soil green algae (Chlorophyta). Systematic Biology 54:936-947.

Maldonado M., Aguilar R., Blanco J., García S., Serrano A., Punzón A. 2015. Aggregated Clumps of Lithistid Sponges: A Singular, Reef-Like Bathyal Habitat with Relevant Paleontological Connections. PloS ONE 10:e0125378.

Maldonado M., Young CM. 1996. Bathymetric patterns of sponge distribution on the Bahamian slope. Deep Sea Research Part I: Oceanographic Research Papers 43:897-915. Marshall W. 1876. Ideen über die Verwandtschaftsverhältnisse der Hexactinelliden. Zeitschrift für wissenschaftliche Zoologie 21:113-136. 
McInerney JO., Adams CI., Kelly M. 1999. Phylogenetic resolution potential of $18 \mathrm{~S}$ and 28S rRNA genes within the lithistid Astrophorida. Memoirs of the Queensland Museum 44:343-351.

Meyer CP., Geller JB., Paulay G. 2005. Fine scale endemism on coral reefs: Archipelagic differentiation in turbinid gastropods. Evolution 59:113-125.

Miloslavich P., Díaz JM., Klein E., Alvarado JJ., Díaz C., Gobin J., Escobar-Briones E., Cruz-Motta JJ., Weil E., Cortés J., Bastidas AC., Robertson R., Zapata F., Martín A., Castillo J., Kazandjian A., Ortiz M. 2010. Marine Biodiversity in the Caribbean: regional estimates and distribution patterns. PLOS ONE 5:e11916.

Moret L. 1926. Contribution à l'étude des spongiaires siliceux du Crétacé superieur français. Mémoires de la Société géologique de France 5:1-327.

Morrow C., Cárdenas P. 2015. Proposal for a revised classification of the Demospongiae (Porifera). Frontiers in Zoology 12:1-27.

Muricy G, and Diaz MC. 2002. Order Homosclerophorida Dendy, 1905. Family Plakinidae Schulze, 1880. In: Hooper JNA, and Van Soest RWM, eds. Systema Porifera A guide to the classification of sponges. New York, Boston, Dordrecht, London, Moscow: Kluwer Academic/ Plenum Publishers, 71-82.

Nardo GD. 1833. De Spongiis. In: Isis, oder Encyclopädische Zeitung Coll. Oken, Jena 519-523.

Pallas PS. 1766. Elenchus zoophytorum sistens generum adumbrationes generaliores et specierum cognitarum succintas descriptiones, cum slectis auctorum synonymis. Fransiscum Varrentrapp, Hagae 451.

Pisera A. 1999. Lithistid sponge Setidium obtectum Schmidt, 1879, rediscovered. In: Hooper JNA ed. Memoirs of the Queensland Museum Proceedings of the 5th International Sponge Symposium, Brisbane, June-July 1998. 473-478.

Pisera A. 2002. Fossil "Lithistids": An Overview. In: Hooper JNA, Van Soest RWM eds. Systema Porifera. A guide to the classification of sponges. New York, Boston, Dordrecht, London, Moscow: Kluwer Academic/ Plenum Publishers, 388-402. Pisera A. 2006. Palaeontology of sponges - a review. Canadian Journal of Zoology 84:242-261.

Pisera A., Lévi C. 2002a. Family Theonellidae Lendenfeld, 1903. In: Hooper JNA, Van Soest RWM eds. Systema Porifera. A guide to the classification of sponges. New York, Boston, Dordrecht, London, Moscow: Kluwer Academic/ Plenum Publishers, 327-337.

Pisera A., Lévi C. 2002b. Family Azoricidae Sollas, 1888. In: Hooper JNA, Van Soest RWM eds. Systema Porifera. A guide to the classification of sponges. New York, Boston, Dordrecht, London, Moscow: Kluwer Academic/ Plenum Publishers, 352-355.

Pisera A., Lévi C. 2002c. Family Scleritodermidae Sollas, 1888. In: Hooper JNA, Van Soest RWM eds. Systema Porifera. A guide to the classification of sponges. New York, Boston, Dordrecht, London, Moscow: Kluwer Academic/ Plenum Publishers, 302-311.

Pisera A., Lévi C. 2002d. Family Corallistidae Sollas, 1888. In: Hooper JNA, Van Soest RWM eds. Systema Porifera. A guide to the classification of sponges. New York, Boston, Dordrecht, London, Moscow: Kluwer Academic/ Plenum Publishers, 312-320.

Pisera A., Pomponi SA. 2015. New data on lithistid sponges from the deep Florida shelf with description of a new species of Theonella. Journal of the Marine Biological Association of the United Kingdom 95:1297-1309.

Plaza A., Gustchina E., Baker HL., Kelly M., Bewley CA. 2007. Mirabamides A-D, Depsipeptides from the Sponge Siliquariaspongia mirabilis that inhibit HIV-1 fusion. Journal of Natural Products 70:1753-1760.

Pomponi SA., Kelly M., Reed JA., Wright EA. 2001. Diversity and bathymetric distribution of lithistid sponges in the tropical western Atlantic region. Bulletin of the Biological Society of Washington 10:344-353. 
Redmond NE., Morrow CC., Thacker RW., Diaz MC., Boury-Esnault N., Cardenas P., Hajdu E., Lobo-Hajdu G., Picton BE., Pomponi SA., Kayal E., Collins AG. 2013. Phylogeny and systematics of Demospongiae in light of new small-subunit ribosomal DNA (18S) Sequences. Integrative and Comparative Biology 53:388-415.

Reed JK., Pomponi SA. 1997. Biodiversity and distribution of deep and shallow water sponges in the Bahamas. Proceedings of the 8th International Coral Reef Symposium 2:1387-1392.

Ronquist F., Teslenko M., van der Mark P., Ayres DL., Darling A., Höhna S., Larget B., Liu L., Suchard MA., Huelsenbeck JP. 2012. MrBayes 3.2: Efficient Bayesian Phylogenetic Inference and Model Choice across a Large Model Space. Systematic Biology 61:539-542.

RStudio Team. 2014. RStudio: Integrated Development for $R$.

Ryan W., Carbotte SM., Coplan JO., O'Hara S., Melkonian A., Arko R., Weissel RA., Ferrini V., Goodwillie A., Nitsche F., Bonczkowski., Zemsky R. 2009. Global Multi-Resolution Topography synthesis. Geochemistry, Geophysics, Geosystems 10:1-9.

Sanders HL. 1968. Marine Benthic Diversity: A Comparative Study. The American Naturalist 102:243-282.

Sará M., Vacelet J. 1973. Écologie des Démosponges. In: Grassé P-P ed. Traité de Zoologie. Anatomie, Systématiqué, Biologie. Paris: Masson et Cie, 472-576.

Schlacher-Hoenlinger MA., Pisera A., Hooper JNA. 2005. Deep-sea "lithistid" assemblages from the Norfolk Ridge (New Caledonia), with description of seven new species and a new genus (Porifera, Demospongiae). Zoosystema 27:649-698.

Schmidt O. 1870. Grundzüge einer Spongien-Fauna des atlantischen Gebietes. Verlag von Wilhelm Engelmann, Leipzig.

Schmidt O. 1879. Die Spongien des Meerbusen von Mexico. I Abt. Lithistiden. Verlag G. Fischer.

Schrammen A. 1910. Neue Kieselschwämme aus der oberen Kreide von Nordwestdeutschland. I. Teil. Tetraxonia, Monaxonia und Silicea incertae sedis. Palaeontographica 5:1-175.

Schrammen A. 1924. Die Kieselspongien der oberen Kreide von Nordwestdeutschland. III. und letzter Teil. Monographien zur Geologie und Paläontologie 1:1-159.

Schuster A., Erpenbeck D., Pisera A., Hooper J., Bryce M., Fromont J., Wörheide G. 2015. Deceptive Desmas: Molecular phylogenetics suggests a new classification and uncovers convergent evolution of lithistid demosponges. PloS ONE 10:e116038.

Schuster A., Lopez JV., Becking LE., Kelly M., Pomponi SA., Wörheide G., Erpenbeck D., Cárdenas P. 2017. Evolution of group I introns in Porifera: new evidence for intron mobility and implications for DNA barcoding. BMC Evolutionary Biology 17:1-21.

Schuster A., Cárdenas, P., Pisera A., Pomponi SA., Kelly M., Wörheide G., Erpenbeck D. 2018. Seven new deep-water Tetractinellida (Porifera: Demospongiae) from the Galápagos Islands - Morphological descriptions and DNA barcodes. Zoological Journal of the Linnean Society 184:1-31.

Sollas WJ. 1887. Sponges. In Black, AC, eds. Encyclopaedia Britannica 412-429.

Sollas WJ. 1888. Report on the Tetractinellida collected by H.M.S. Challenger, during the years 1873-1876. Zoology 25:1-458.

Stamatakis A. 2014. RAxML version 8: a tool for phylogenetic analysis and post-analysis of large phylogenies. Bioinformatics 30:1312-1313.

Tautz D., Renz M. 1983. An optimized freeze-squeeze method for the recovery of DNA fragments from agarose gels. Analytical Biochemistry 132:14-19.

Topsent E. 1894. Etude monographique des spongiares de France. I. Tetractinellida. Archives de Zoologie Expérimentale et Générale 3:259-400. 
Van Soest RWM., Boury-Esnault N., Hooper JNA., Rützler K., de Voogd NJ., Alvarez B., Hajdu E., Pisera AB., Manconi R., Schönberg C., Klautau M., Picton B., Kelly M., Vacelet J., Dohrmann M., Díaz, MC., Cárdenas P., Carballo JL., Ríos P., Downey R. 2018b. World Porifera database. Corallistes Schmidt, 1870. Accessed at: http://www.marinespecies.org/porifera/porifera.php?p=taxc on 2018-09-20

Van Soest RWM., Boury-Esnault N., Hooper JNA., Rützler K., de Voogd NJ., Alvarez B., Hajdu E., Pisera AB., Manconi R., Schönberg C., Klautau M., Picton B., Kelly M., Vacelet J., Dohrmann M., Díaz, MC., Cárdenas P., Carballo JL., Ríos P., Downey R. 2018a. World Porifera database. Macandrewia Gray, 1859. Accessed at: http://www.marinespecies.org/porifera/porifera.php?p=taxd on 2018-09-20

Van Soest RWM., Boury-Esnault N., Hooper JNA., Rützler K., de Voogd NJ., Alvarez B., Hajdu E., Pisera AB., Manconi R., Schönberg C., Klautau M., Picton B., Kelly M., Vacelet J., Dohrmann M., Díaz, MC., Cárdenas P., Carballo JL., Ríos P., Downey R. 2018c. World Porifera database. Neophrissospongia Pisera and Lévi, 2002. Accessed at: http://www.marinespecies.org/porifera/porif on 2018-09-20

Van Soest RWM., Hooper JNA. 2002. Order Spirophorida Bergquist and Hogg, 1969. In: Hooper JNA, Van Soest RWM eds. Systema Porifera. A guide to the classification of sponges. New York, Boston, Dordrecht, London, Moscow: Kluwer Academic/ Plenum Publishers, 83-84.

Van Soest RWM., Stentoft N. 1988. Barbados deep-water sponges. Studies on the Fauna of Curaçao and Other Caribbean Islands 70:1-175.

Vargas S., Kelly M., Schnabel K., Mills S., Bowden D., Wörheide G. 2015. Diversity in a cold hot-spot: DNA-barcoding reveals patterns of evolution among antarctic demosponges (Class Demospongiae, Phylum Porifera). PloS ONE 10:e0127573.

von Lendenfeld R. 1903. Tetraxonia. In Schulze FE eds. Das Tierreich. Friedländer, Berlin Publishers, 1-168.

Wickham H. 2009. ggplot2: Elegant Graphics for Data Analysis. New York: Spinger-Verlag.

Wright AE. 2010. The Lithistida: important sources of compounds useful in biomedical research. Current Opinion in Biotechnology 21:801-807.

Xia X. 2013. DAMBE5: a comprehensive software package for data analysis in molecular biology and evolution. Molecular Biology and Evolution 30:1720-1728.

Xia X., Xie Z., Salemi M., Chen L., Wang Y. 2003/1. An index of substitution saturation and its application. Molecular Phylogenetics and Evolution 26:1-7. 


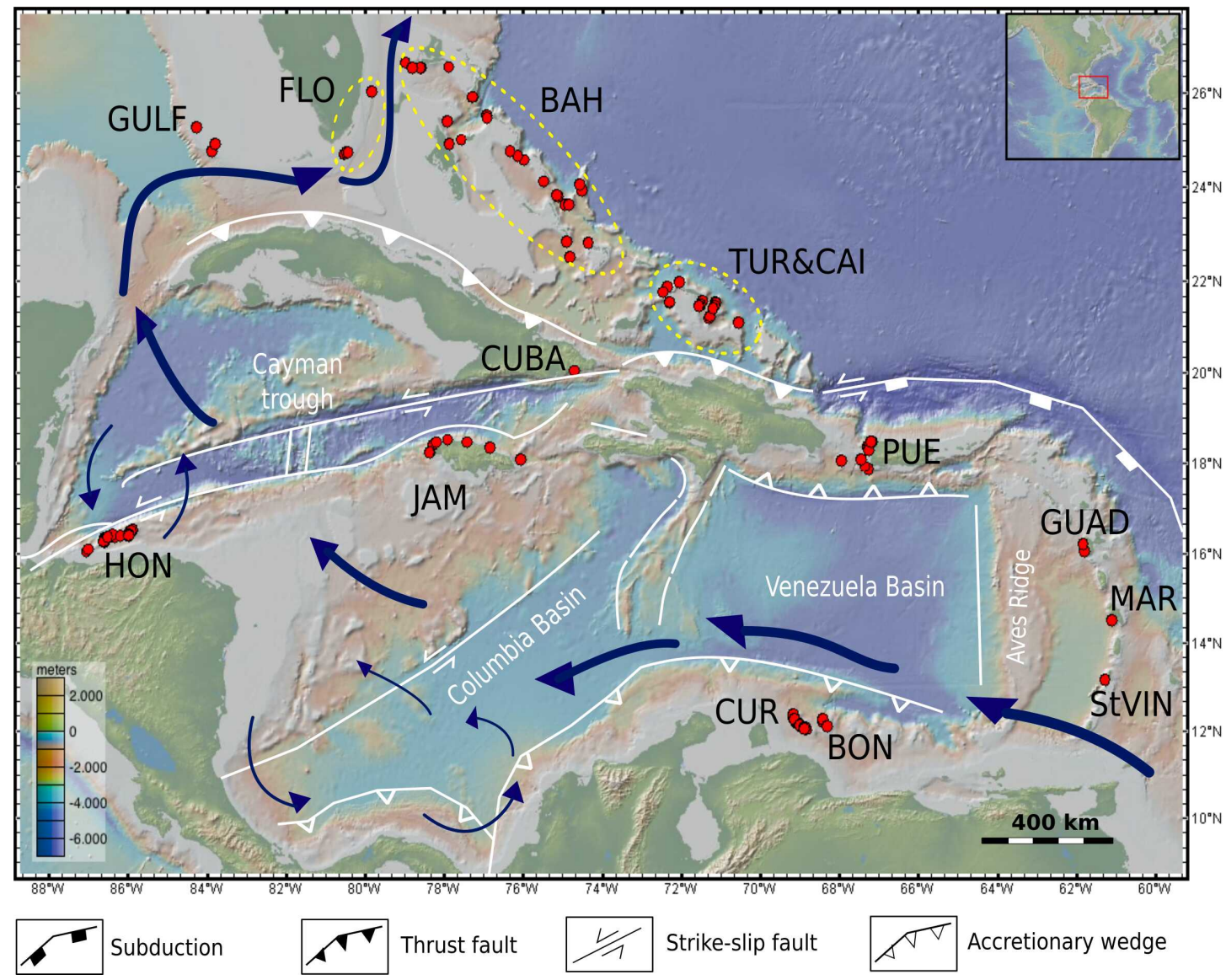

Figure 1. Distribution map of investigated HBOI and other desma-bearing tetractinellids and Vetulinidae from the TWA. Abbreviations correspond to the different locations (CUR=Curaçao, BON=Bonaire, StVIN=St. Vincent, MAR=Martinique, GUAD=Guadaloupe, PUE=Puerto Rico, JAM=Jamaica, HON=Honduras, TUR and CAI=Turks and Caicos, BAH=Bahamas, FLO=Florida). Tectonic settings are schematically indicated (white lines) (Giunta 2011). Main water currents (dark blue arrows) follow Miloslavich et al. (2010). Map generated with GeoMapApp 3.6.3 (http://www.geomapapp.org, Ryan et al., 2009). 


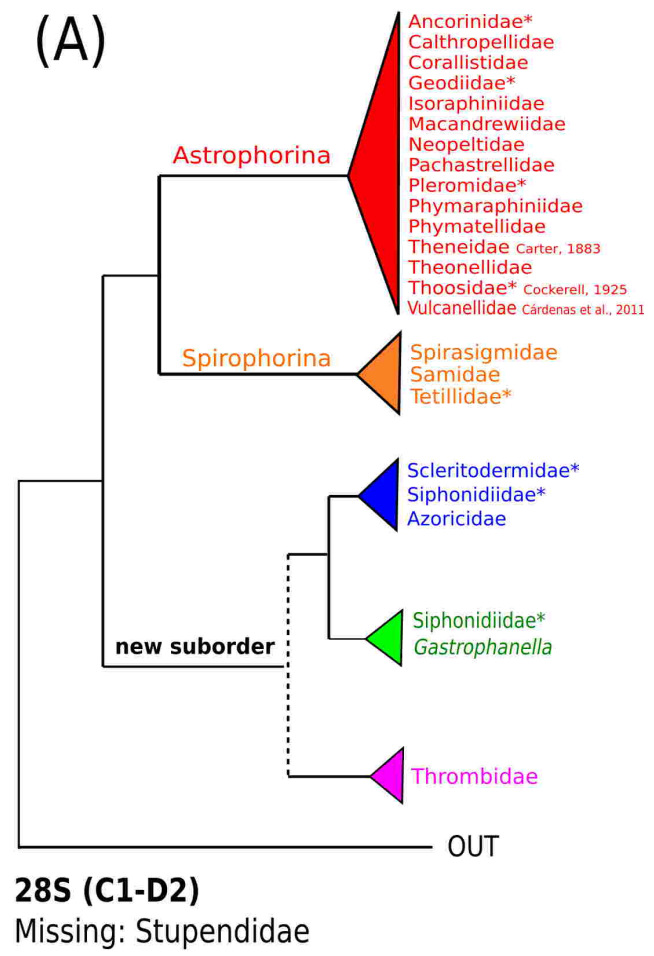

(B)
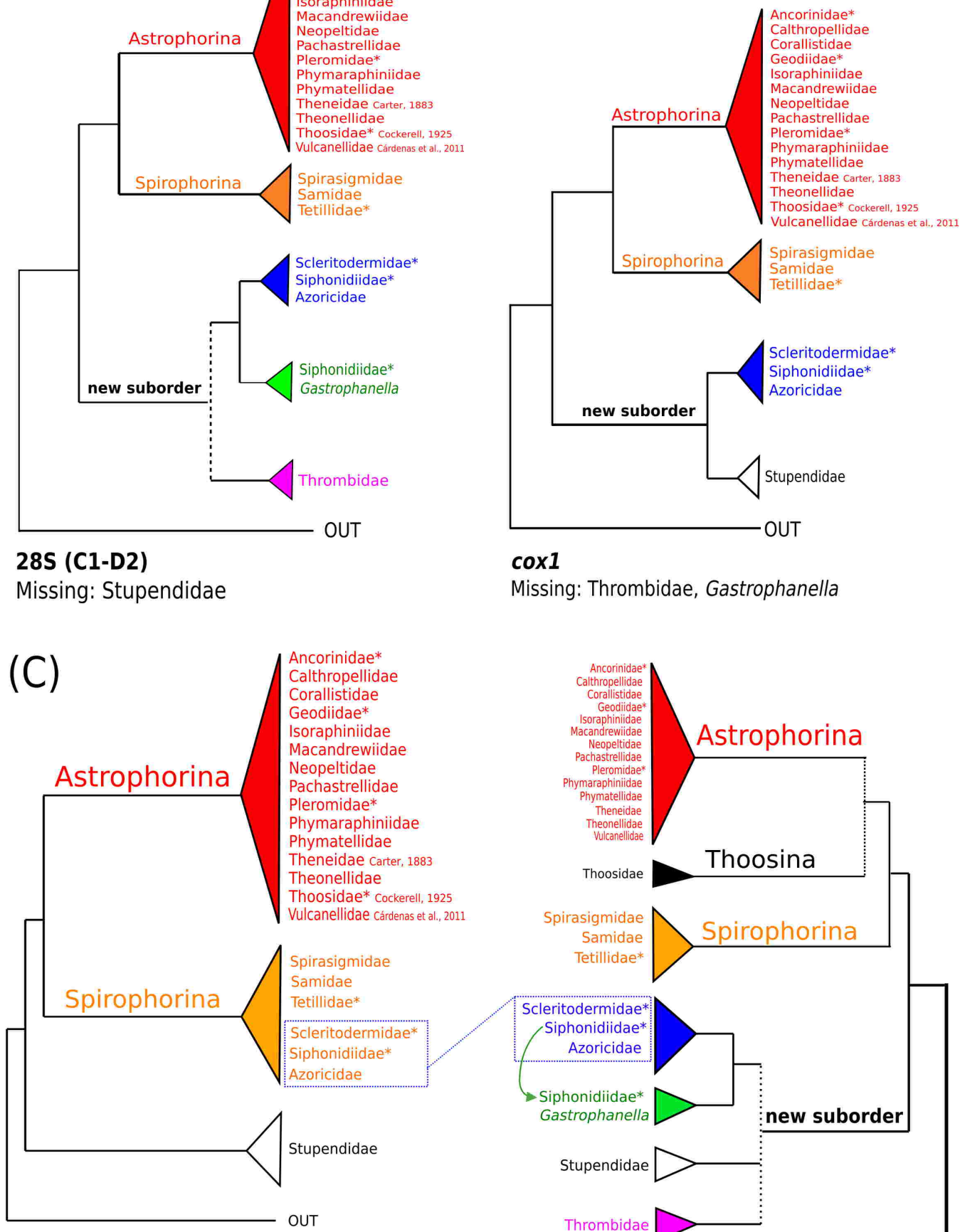

Current classification

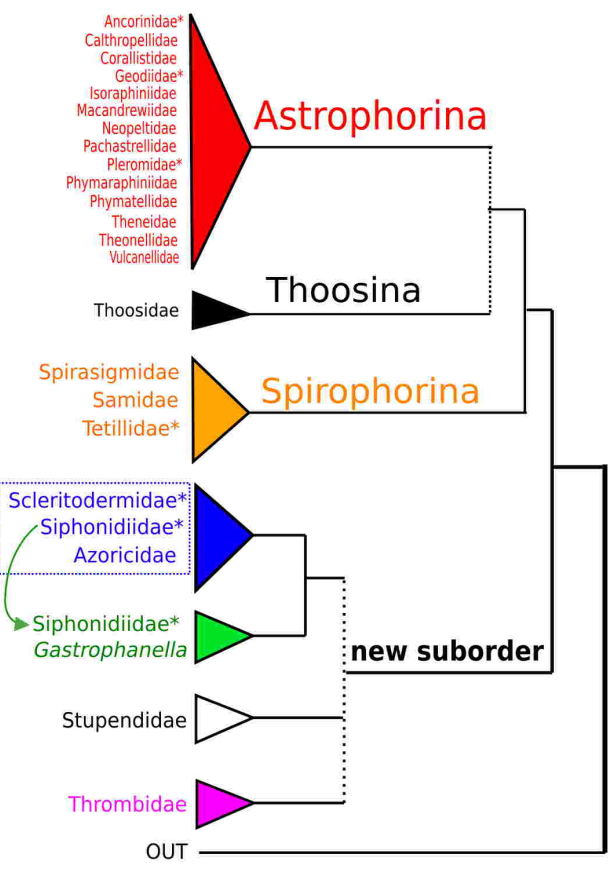

Our revised classification

Figure 2. Schematic summary cladograms obtained from the $28 \mathrm{~S}$ and cox 1 phylogenies indicating the higher-taxa relationships within the order Tetractinellida. (A) 28S (B) cox 1 summary tree with the suborders Astrophorina (red), Spirophorina (orange) and a new suborder (blue, green, pink and light gray) including all rhizoclone desma-bearing families and the family Thrombidae and Stupendidae. Stars behind family names indicate their proposed polyphyly. Dashed lines indicate the uncertainties of not supported topologies. (C) Comparison of current and revised classification including the proposed new suborder Thoosina from Carballo et al. 


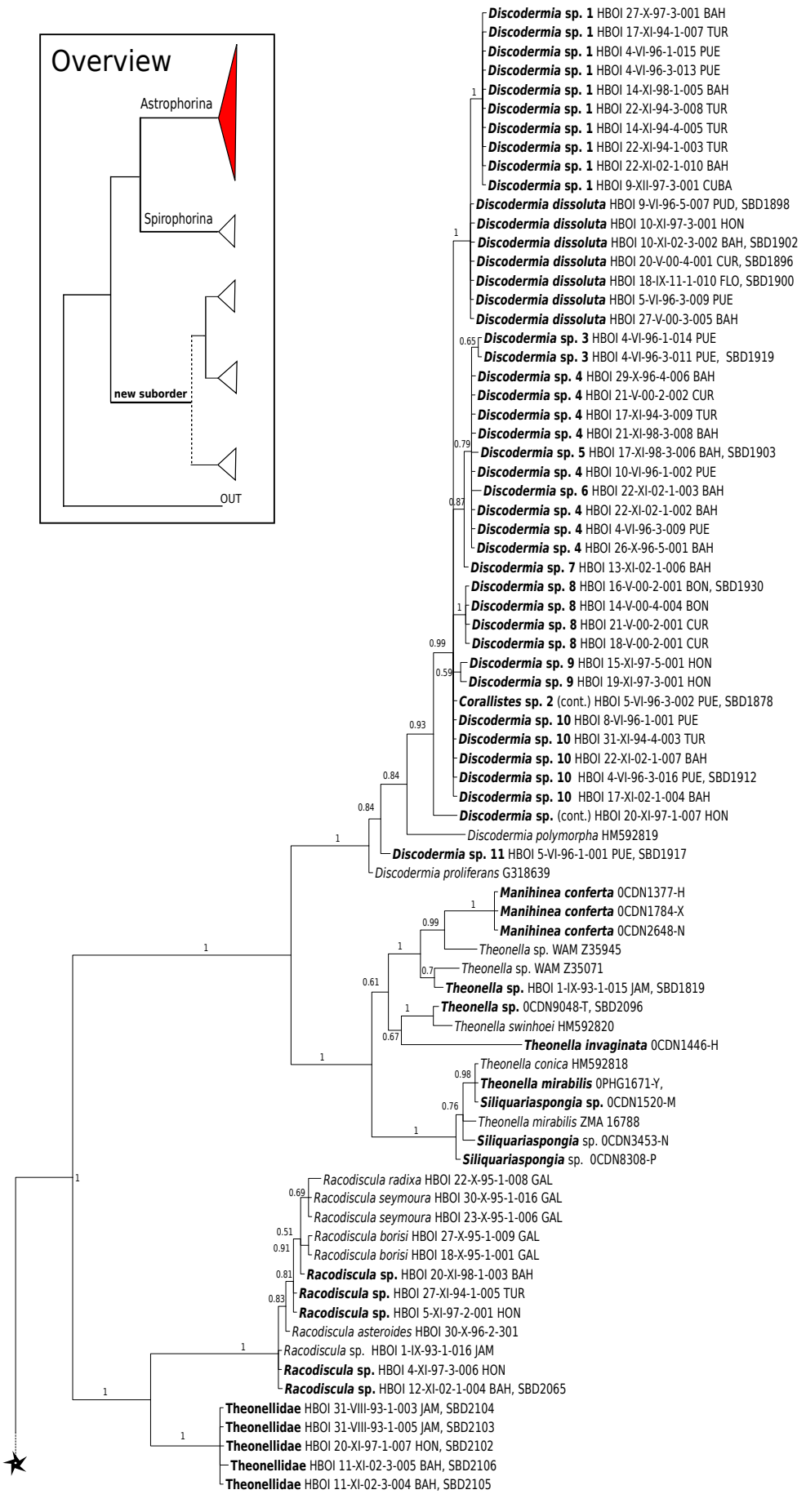

0.1 


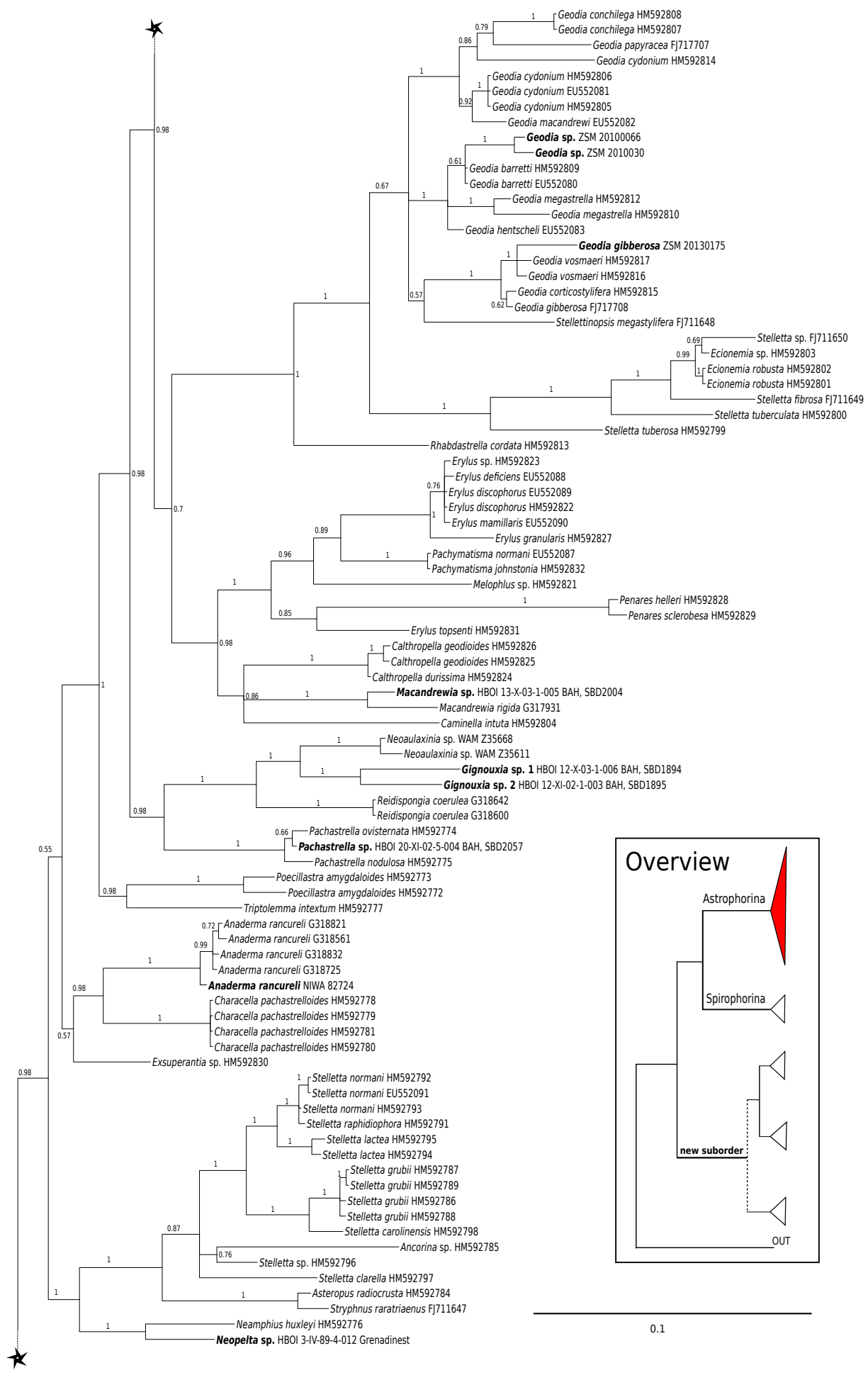




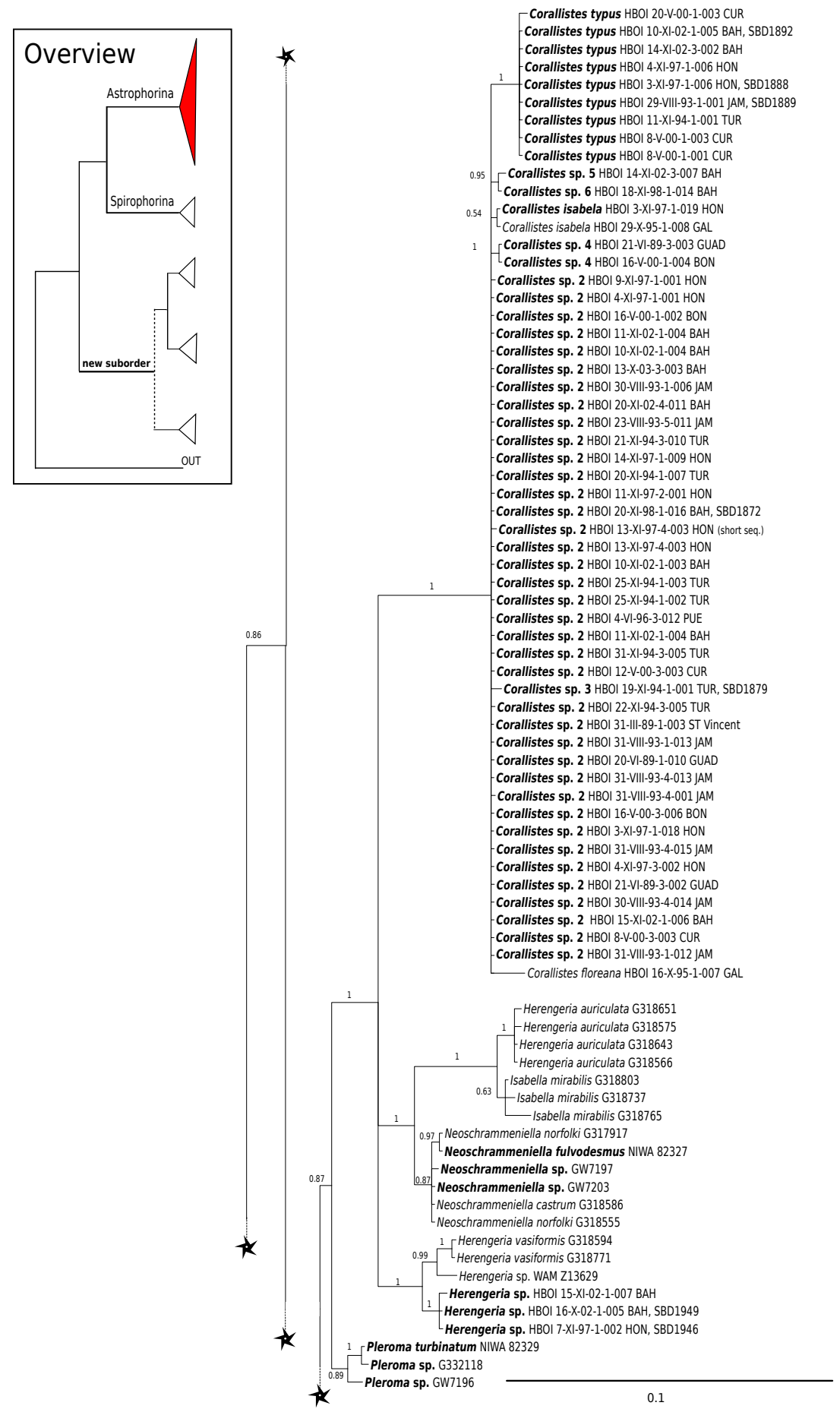




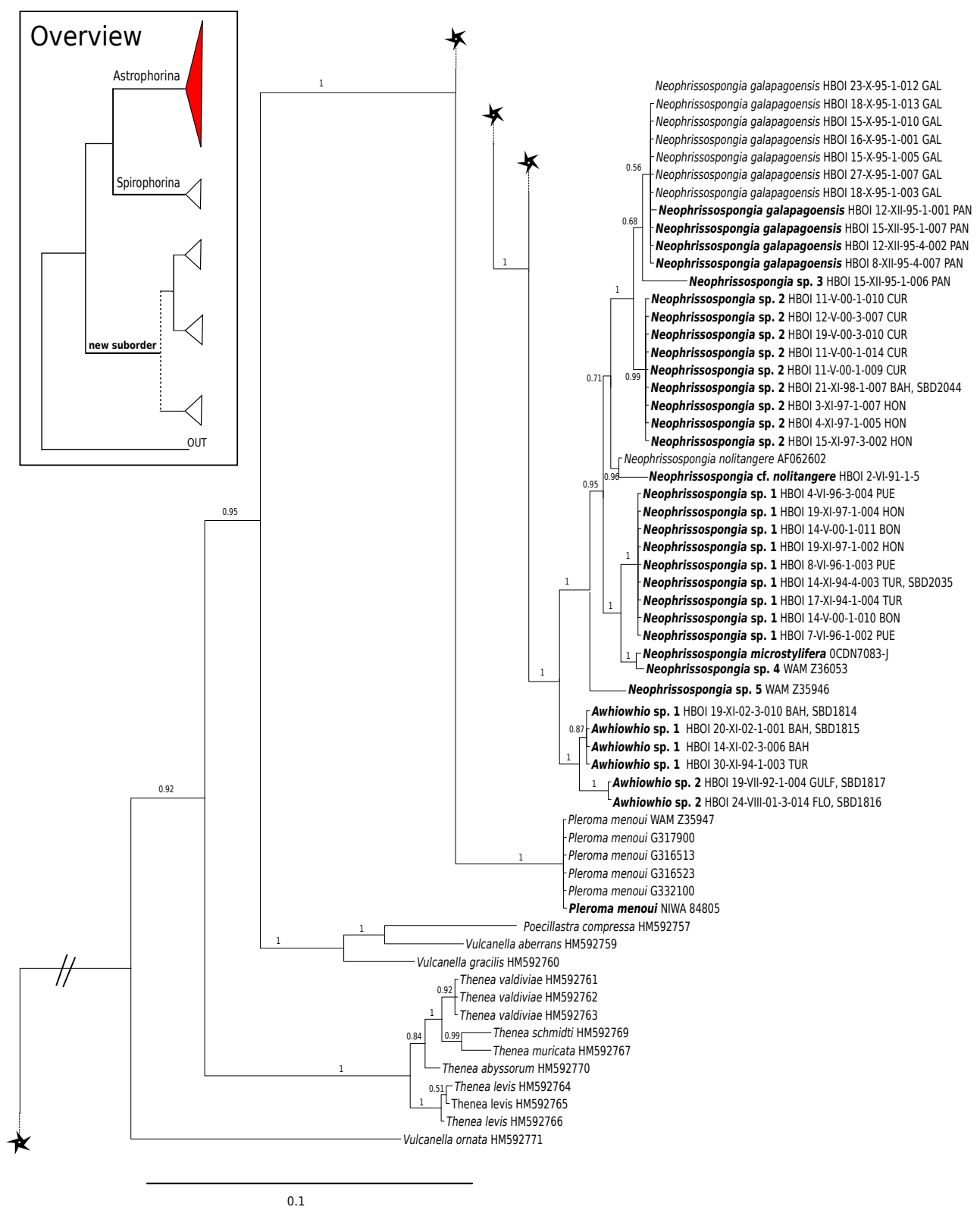




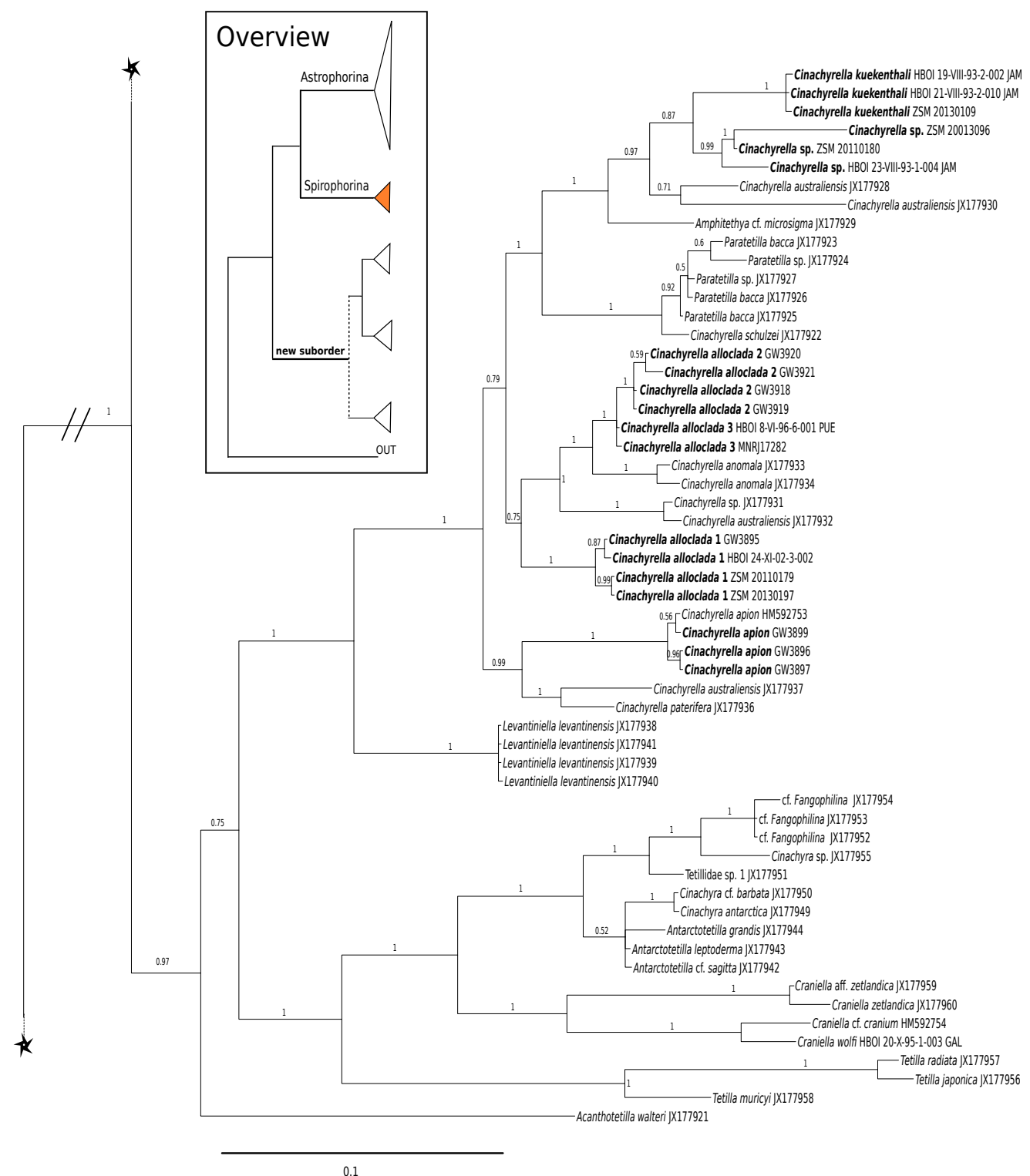




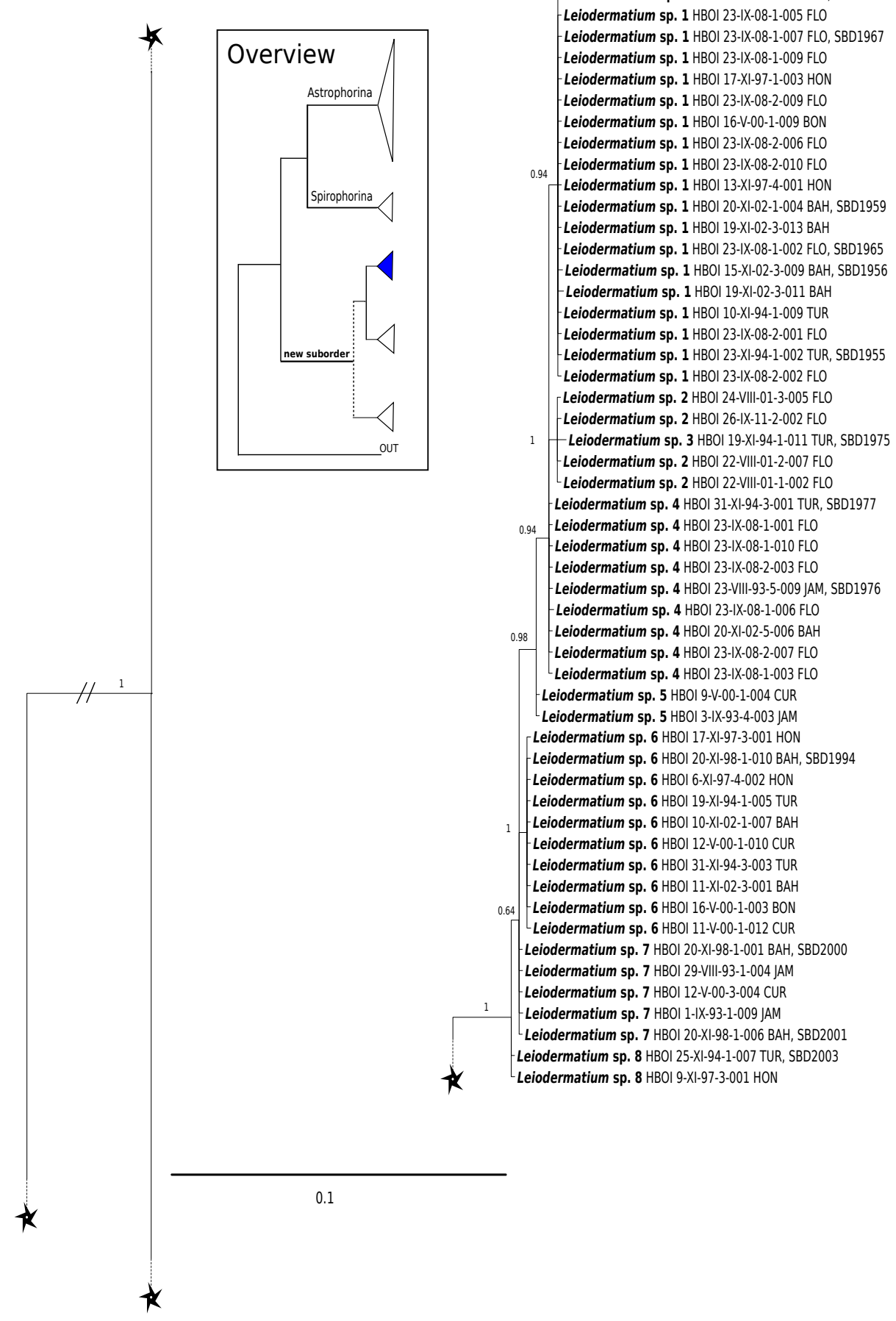

-Leiodermatium sp. 1 HBOI 23-IX-08-1-004 FLO, SBD1966 eiodermatium sp. $1 \mathrm{HBO}$ 23-IX-08-1-005 FLO

e. 1 HBOI $23-1 X-08-1-007$ FLO, SBD1967 Leiodermatium sp. 1 HBO 1

Leiodermatium sp. $1 \mathrm{HBO}$ 23-IX-08-2-009 FLO Leiodermatium sp. 1 HBOI 20-XI-02-1-004 BAH, SBD1959 Leiodermatium sp. $1 \mathrm{HBOI}$ 19-XI-02-3-013 BAH (1) 1 HBOI 23-IX-08-1-002 FLO, SBD1965 Leiodermatium sp. $1 \mathrm{HBO}$ 19-XI-02-3-011 BAH

. iodermatium sp. 4 HBOI 31-XI-94-3-001 TUR, SBD1977

-Leiodermatium sp, 4 HBO 23-IX-08-1-001 FLO

Leiodermatium sp. 4 HBOI 23-IX-08-1-010 FLO

Leiodermatium sp. 4 HBOI 23-VIIL-93-5-009 JAM, SBD1976

Leiodermatium sp. $4 \mathrm{HBO}$ 23-IX-08-1-006 FLO Leiodermatium sp. $6 \mathrm{HBO}$ 6-X--97-4-002 HON Leiodermatium sp. 6 HBOI 19-XI-94-1-005 TUR Leiodermatium sp. 6 HBOI 10-XI-02-1-007 BAH matium sp. 6 HBOI 12-V-00-1-010 CUR - Leiodermatium sp. - Leiodermatium sp. 6 HBOI 11-V-00-1-012 CUR eiodermatium sp. 7 HBOI 20-XI-98-1-001 BAH, SBD2000 Lermatium sp. 7 HBOL 29-VIII-93-1-004 JAM Leiodermatium Sp. 7 HBOI 20-XI-98-1-006 BAH, SBD2001 eiodermatium sp. 8 HBOI 25-XX-94-1-007 TUR, SBD2003 


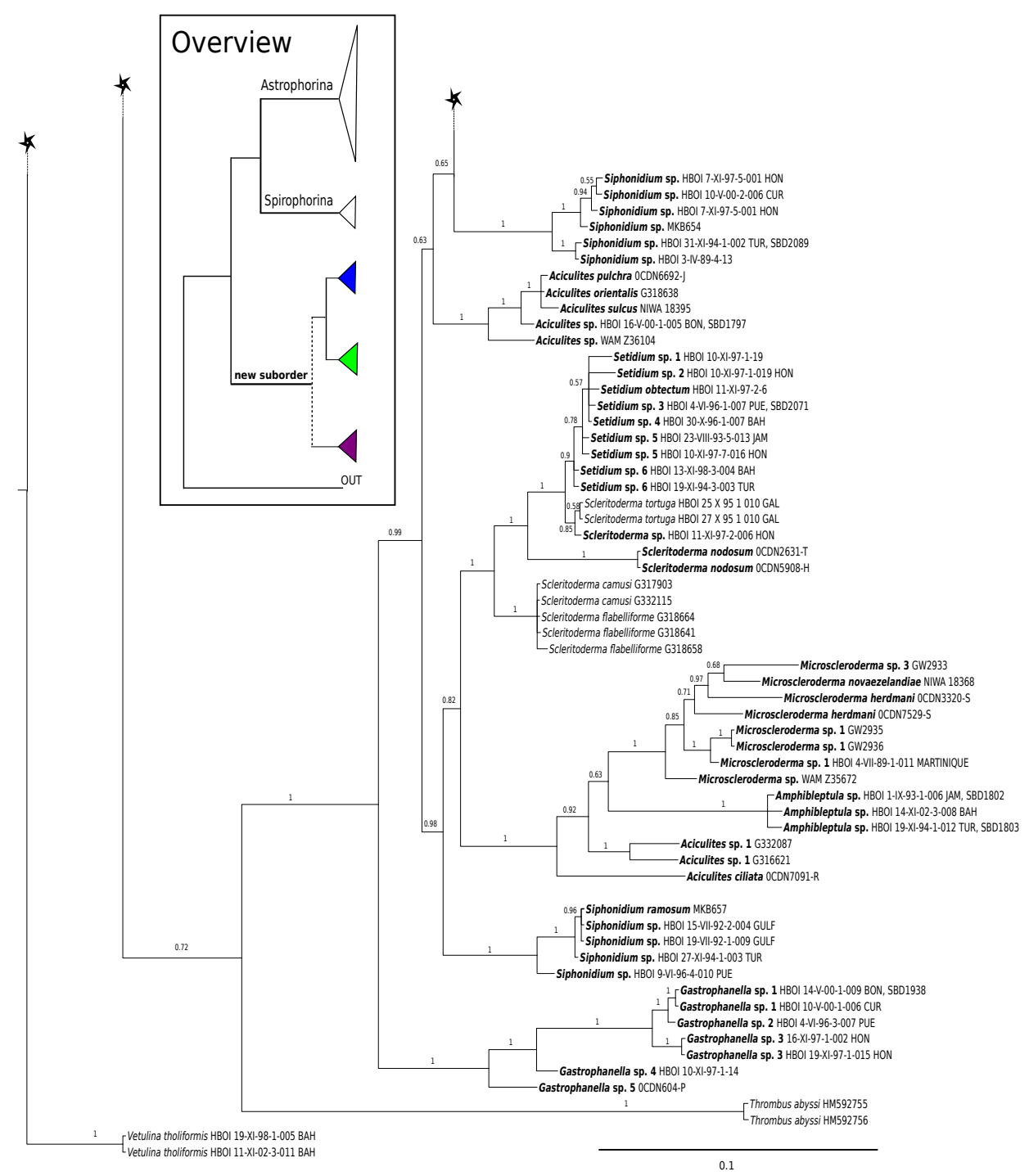

Figure 3. Bayesian Inference phylogeny of Tetractinellida based on 28S (C1-D2). Posterior probability (PP) values are provided above or below branches. Self-generated sequences are in bold. Numbers behind taxon names are either voucher numbers or GenBank/ENA accession numbers. Three letter code behind voucher numbers corresponds to the different locations (CUR=Curaçao, BON=Bonaire, StVIN=St. Vincent, MAR=Martinique, GUAD=Guadaloupe, PUE=Puerto Rico, JAM=Jamaica, HON=Honduras, TUR \& CAI=Turks \& Caicos, $\mathrm{BAH}=$ Bahamas, $\mathrm{FLO}=$ Florida). Taxa where the morphology was investigated are indicated with

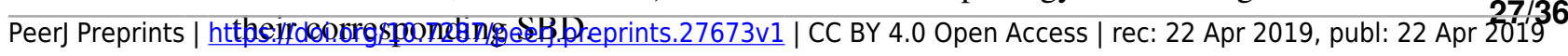




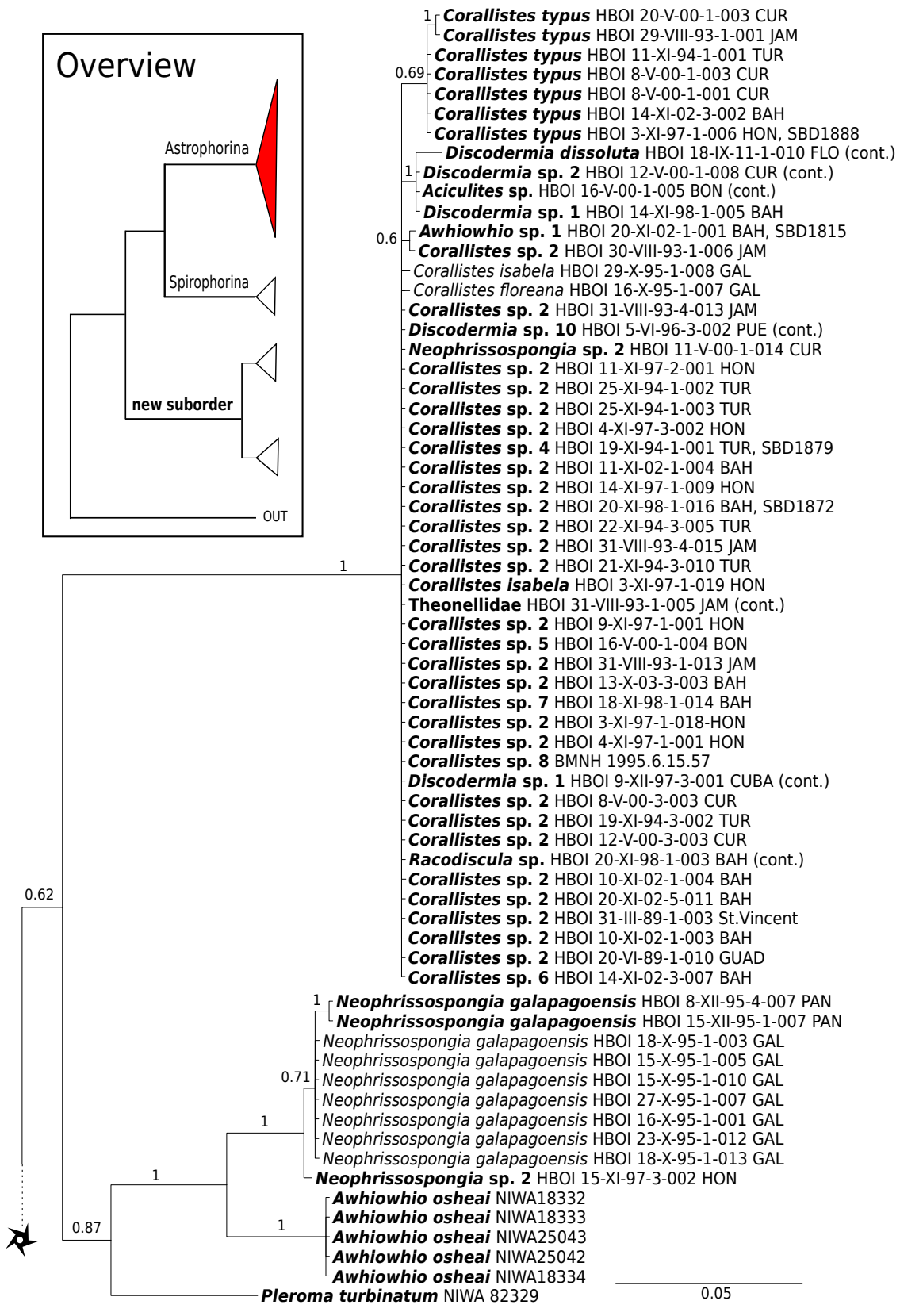




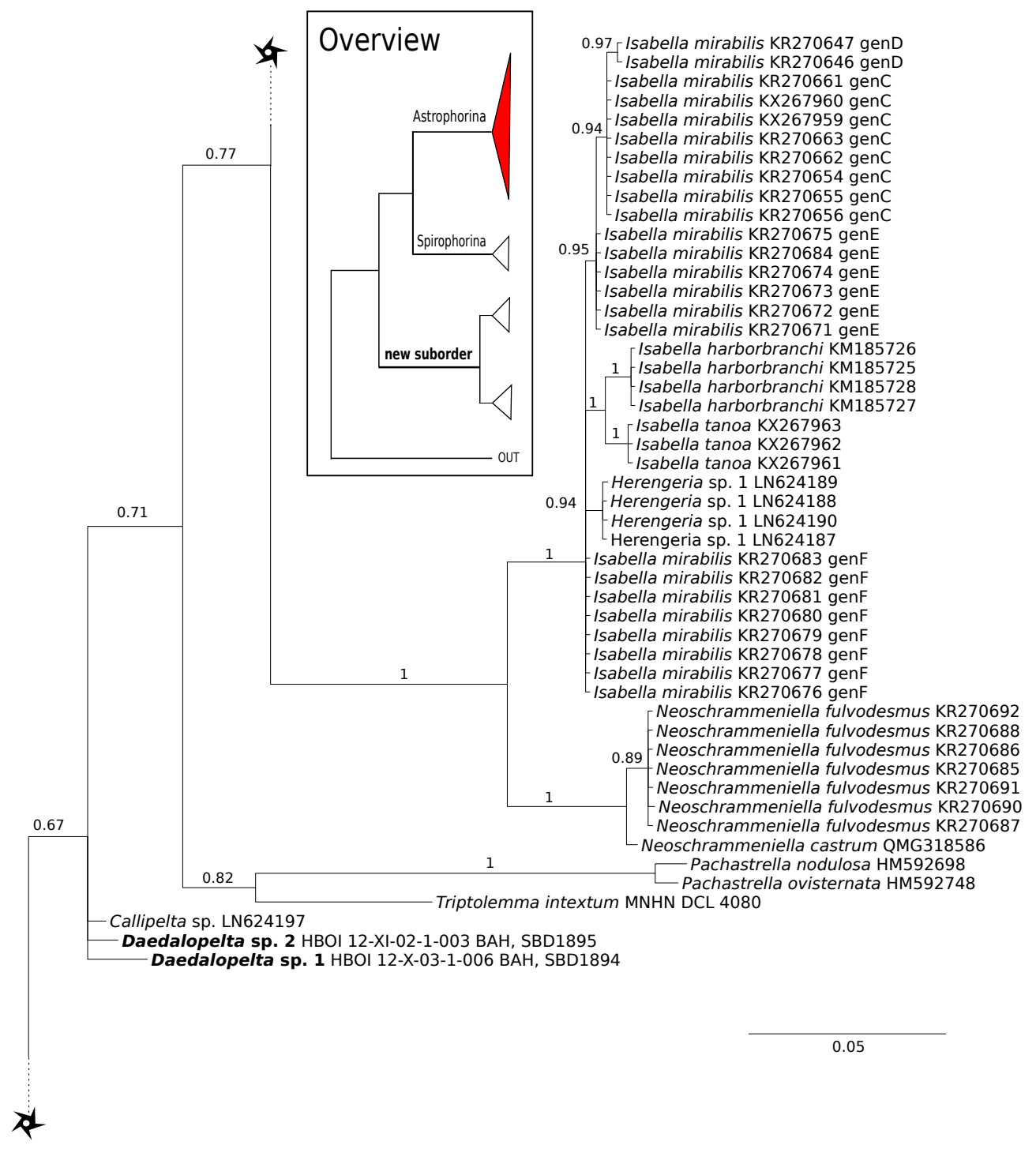




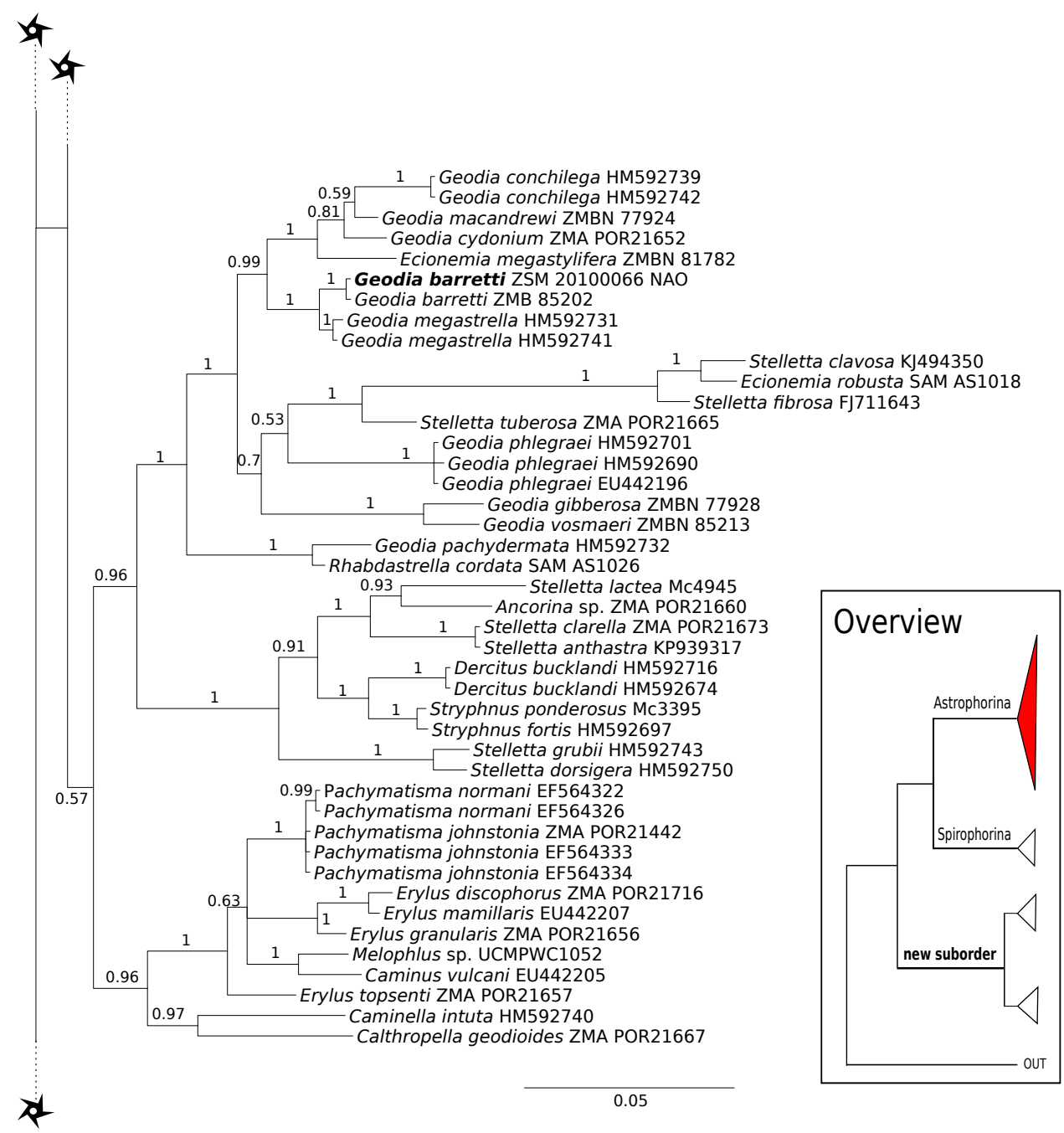




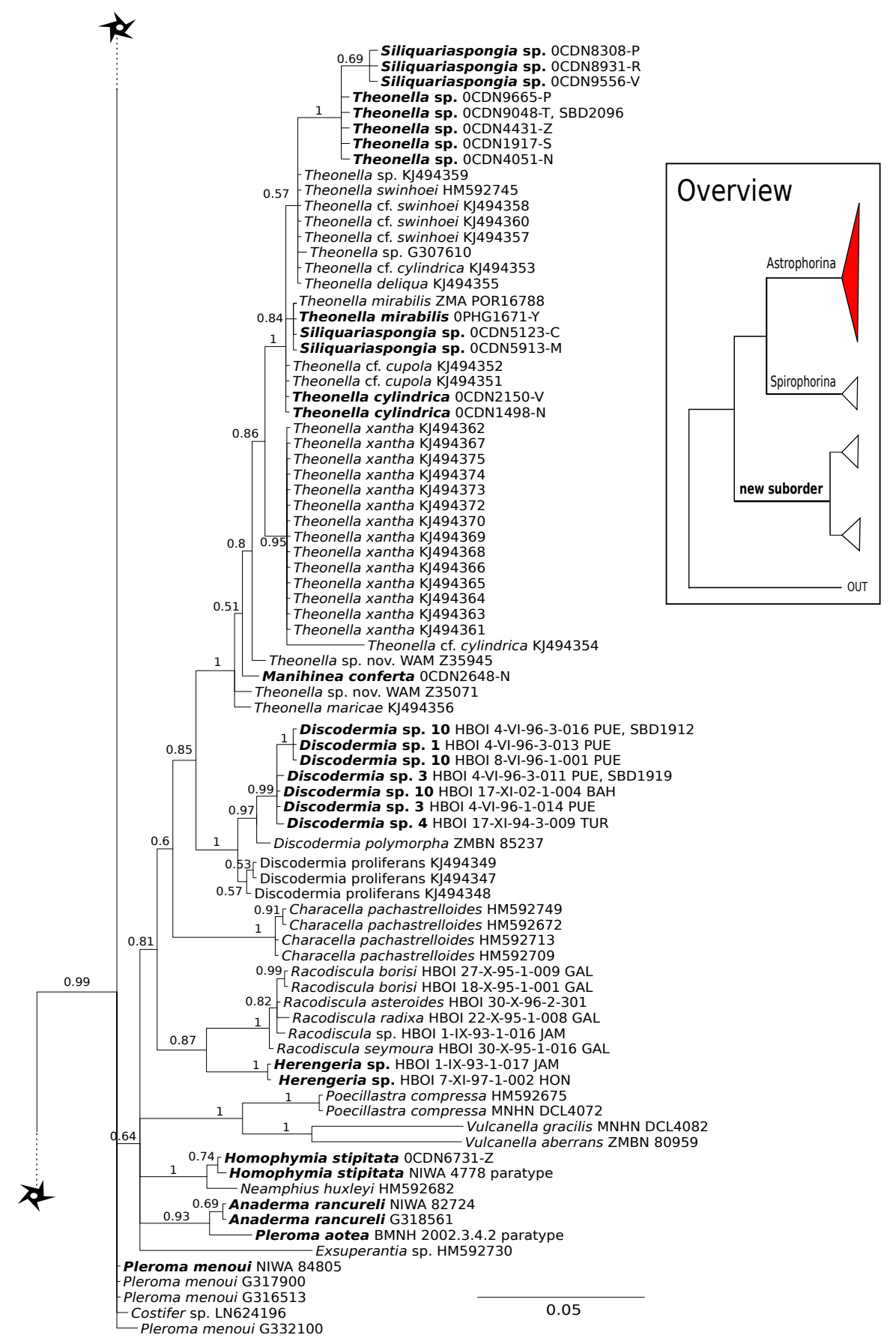




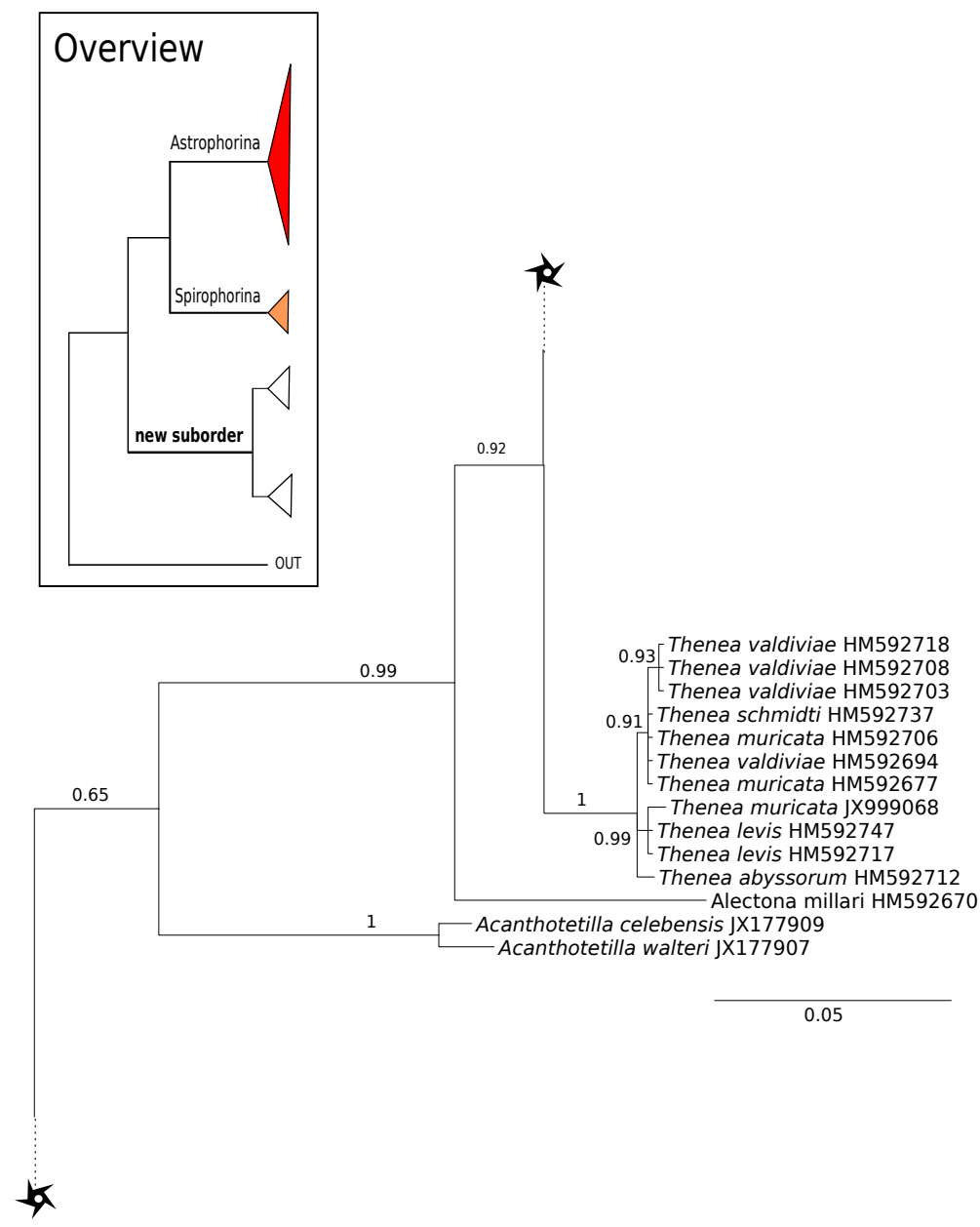




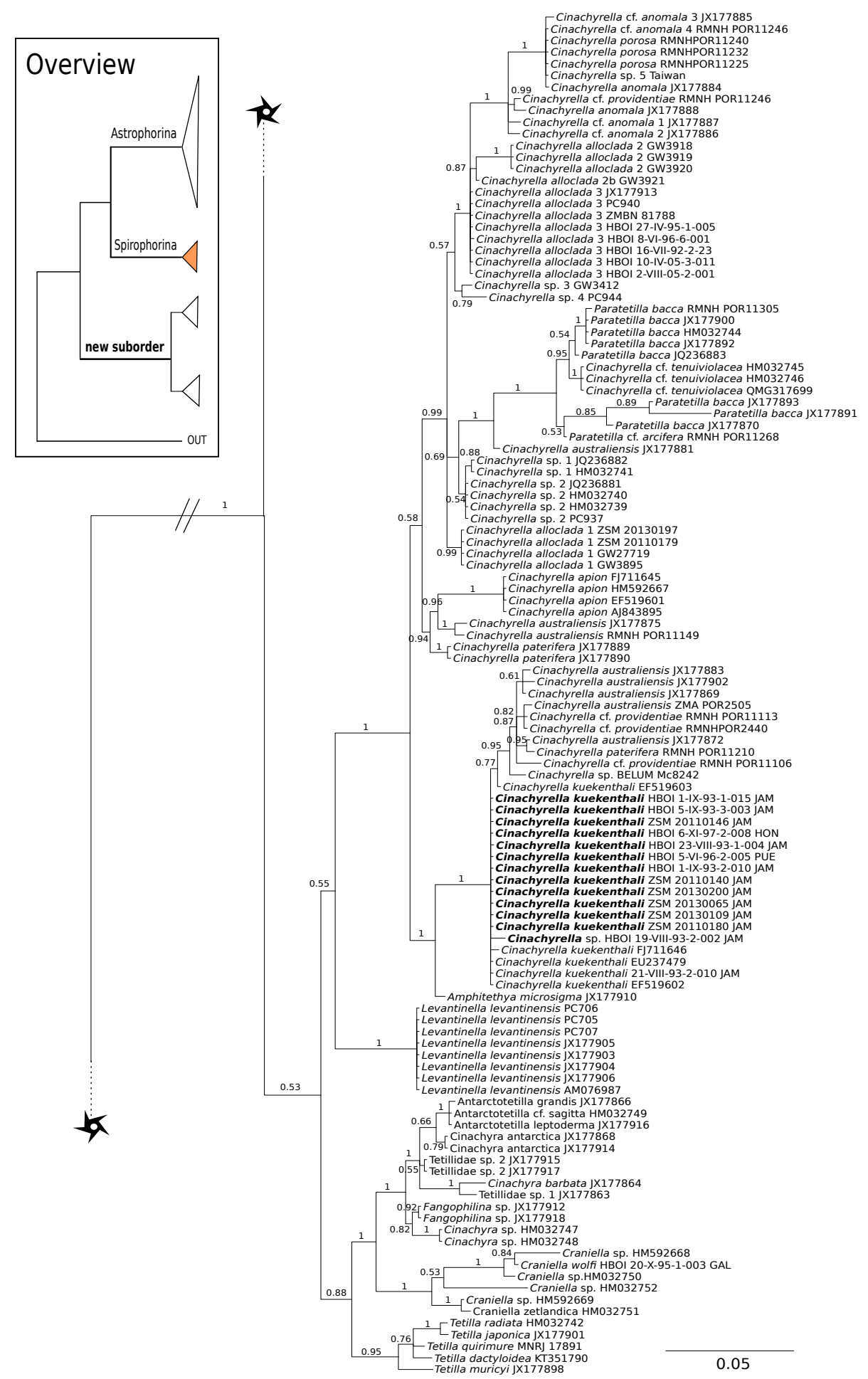




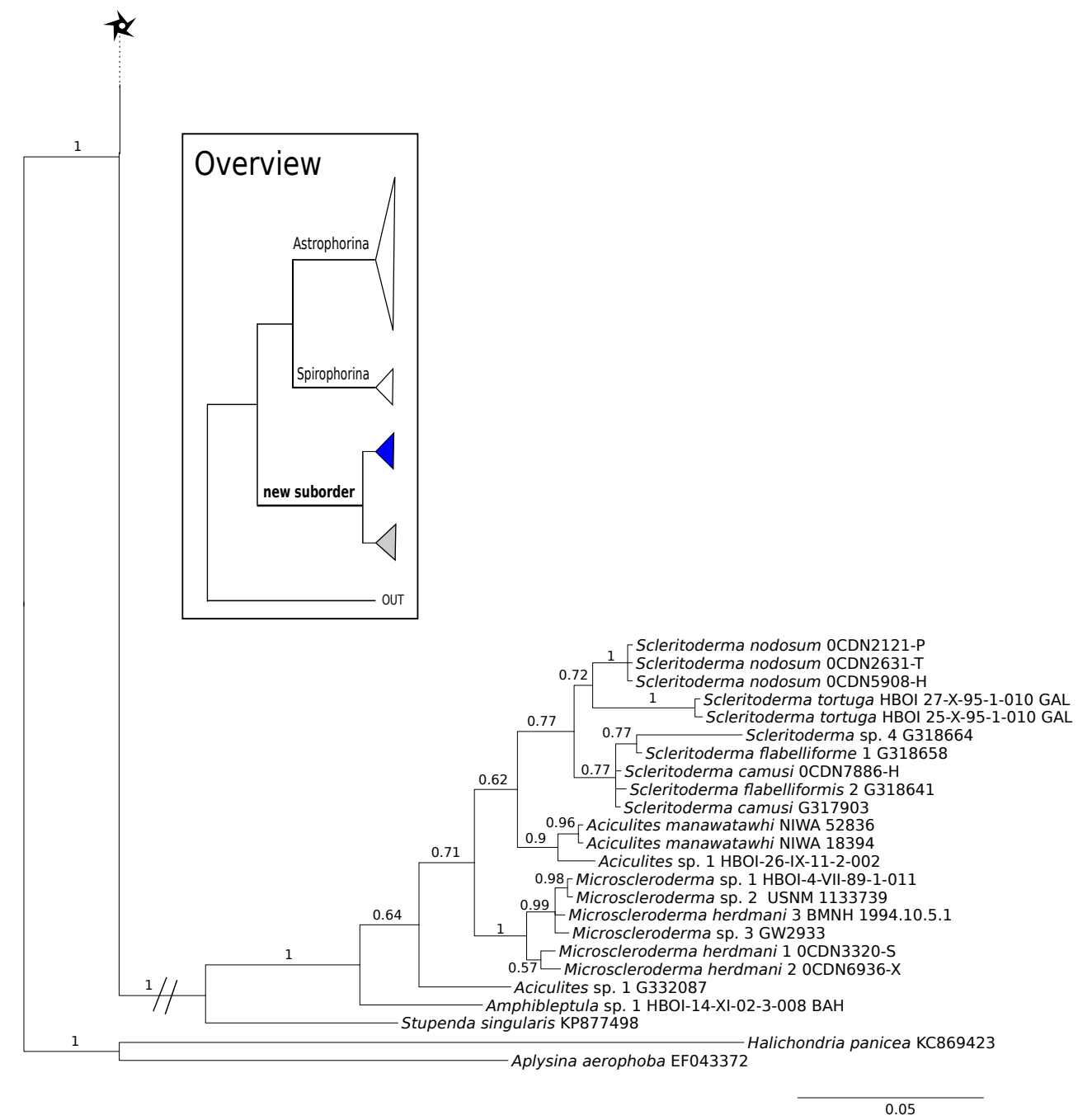

Figure 4. Bayesian Inference phylogeny of Tetractinellida based on cox1. Posterior probability (PP) values are provided above or below branches. Self-generated sequences are in bold. Numbers behind taxon names are either voucher numbers or GenBank/ENA accession numbers. Three letter code behind voucher numbers corresponds to the different locations (CUR=Curaçao, BON=Bonaire, StVIN=St. Vincent, MAR=Martinique, GUAD=Guadaloupe, PUE=Puerto Rico, JAM=Jamaica, HON=Honduras, TUR \& CAI=Turks \& Caicos, BAH=Bahamas, FLO=Florida). Taxa where the morphology was investigated are indicated with their corresponding SBD. 

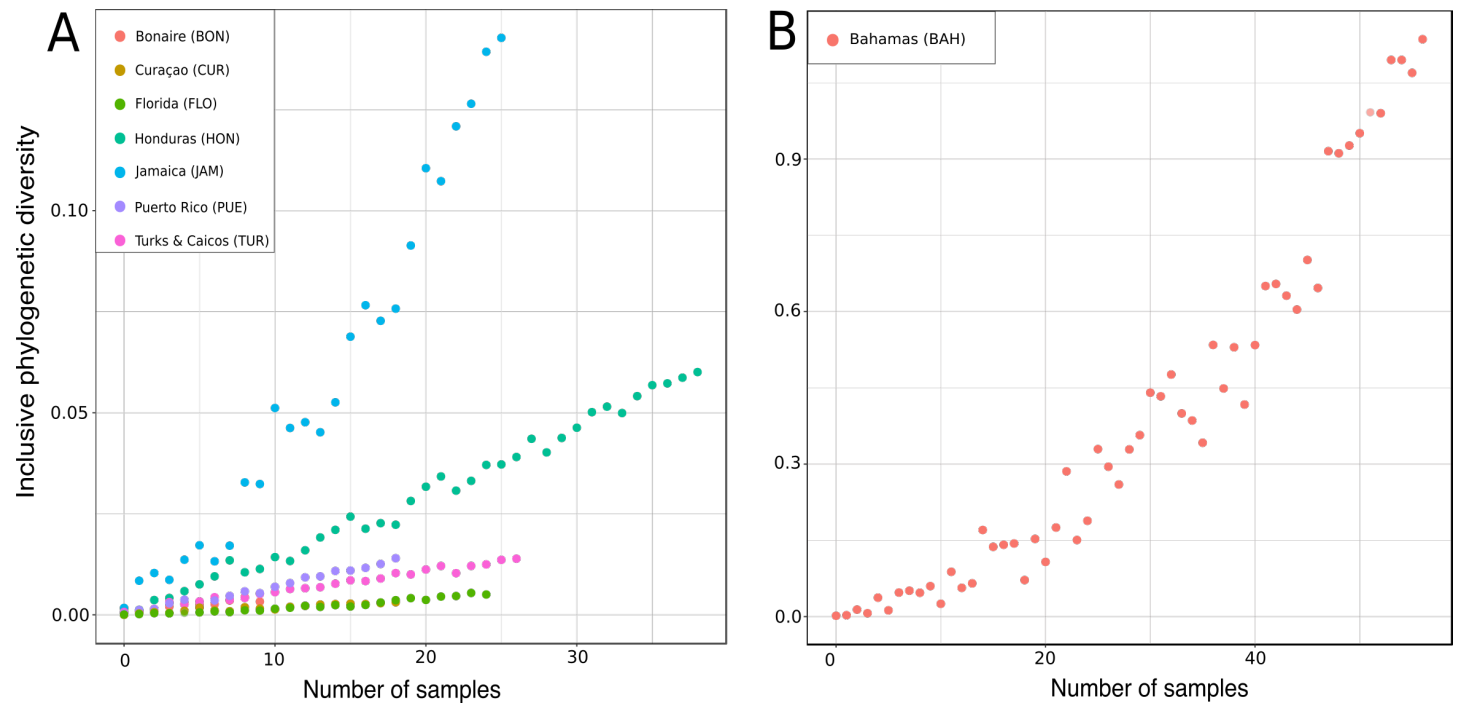

Figure 5. Rarified inclusive phylogenetic diversity $\left(\mathrm{PD}_{\mathrm{I}}\right)$ curves per marine regions analyzed. For a better visualization the PDI for the Bahamas are illustrated separately (B) due to their larger number of samples. 


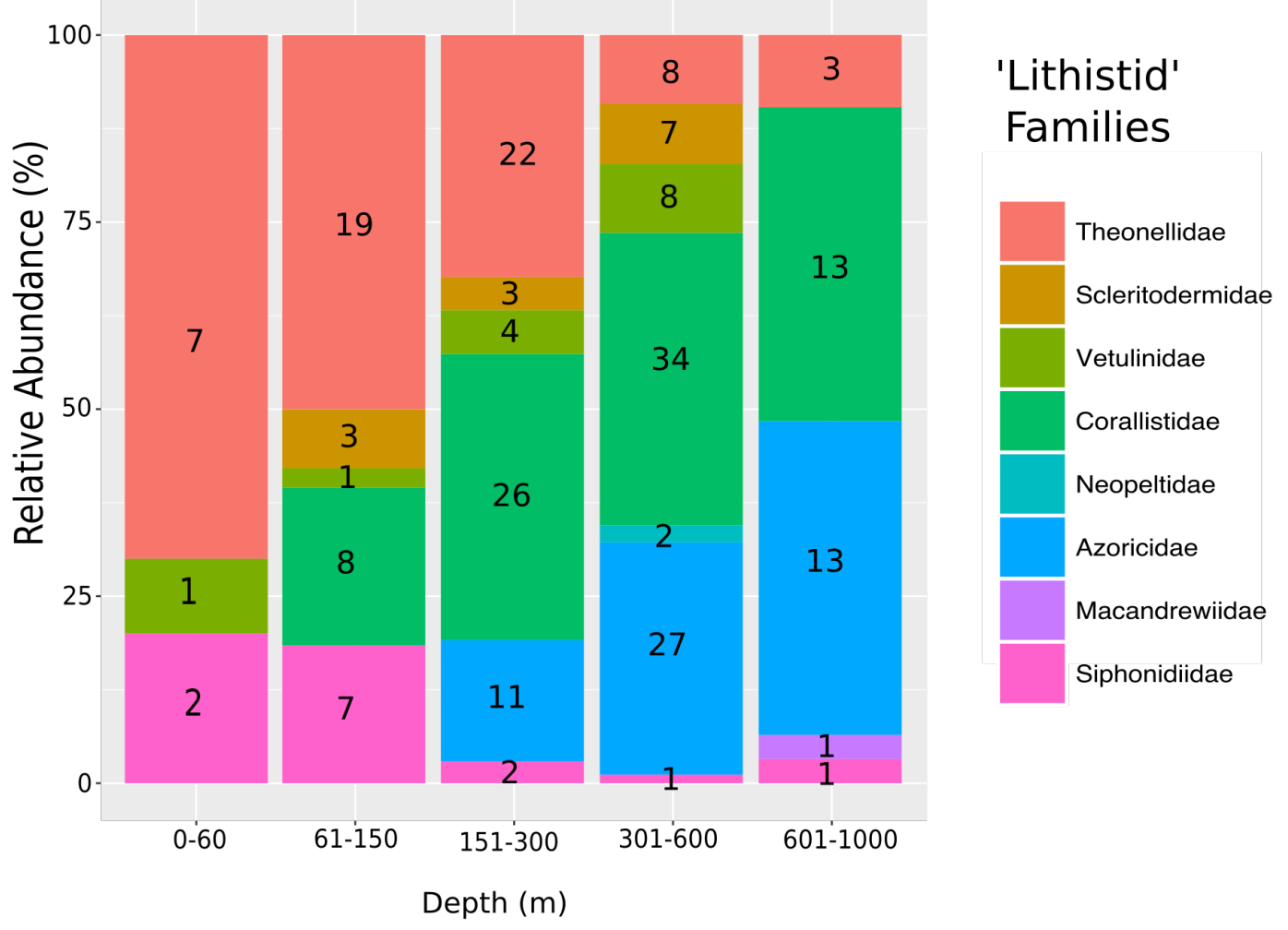

\section{Geomorphological characterizations}

\section{Fore reef escarpment and slope $(0-60 \mathrm{~m})$}

caves, rocky fore reef slope, overhanging rocks.

\section{Deep fore reef escarpment and slope $(61-150 \mathrm{~m})$}

steep fore reef, rocky slopes, vertical walls, crevices, caves, overhanging buttresses.

\section{Island slopes (151-300 m)}

Steep $\left(45-70^{\circ}\right)$ rokcy slopes, often covered with sediment or rubble or 1-5 $\mathrm{m}$ high boulders.

Deep island slope (301-1000 $\mathrm{m})$

Highly variable among sites; fine mud and sand slopes, rocky pinnacles and boulders, vertical walls.

Figure 6. Bathymetric distribution and relative abundance of TWA desma-bearing demosponges based on 234 samples of eight families. Numbers in each bar represent the number of samples investigated. The following genera for each family were included: Leiodermatium (Azoricidae); Corallistes, Herengeria, Neophrissospongia and Awhiowhio (Corallistidae); Macandrewia (Macandrewiidae); Daedalopelta and Neopelta (Neopeltidae); Aciculites, Amphibleptula, Microscleroderma, Scleritoderma and Setidium (Scleritodermidae); Gastrophanella and Siphonidium (Siphonidiidae); Discodermia, Racodiscula and Theonella (Theonellidae); Vetulina (Vetulinidae). Geomorphological characterizations of depth zones are given below the graph and follows Pomponi et al. 2001 and Reed and Pomponi (1997; 2001). 